\title{
The Lifted Root Number Conjecture for some cyclic extensions of $\mathbb{Q}$
}

by

Jürgen Ritter (Augsburg) and Alfred Weiss (Edmonton, Alta.)

1. Introduction. This paper gives first evidence for the Lifted Root Number Conjecture [GRW1] which refines Chinburg's Root Number Conjecture $[\mathrm{Ct}]$. The general setting has also been described in [GRW2]. Here, we observe that the root number conjecture in its lifted form makes predictions about the relations between the global units and the ideal class group which go beyond what Euler systems or the Main Conjecture of Iwasawa theory are known to imply $\left({ }^{1}\right)$.

This is discussed in the simplest case, namely when $K / \mathbb{Q}$ is a cyclic extension of odd prime degree $l$ and squarefree conductor $n=p_{1} \ldots p_{r}$ with all primes $p_{j} \neq l$. Note that $K \subset \mathbb{Q}\left(\zeta_{n}\right)$ where, for a natural number $m, \zeta_{m}$ always denotes a primitive $m$ th root of unity.

Let $G=\left\langle g_{0}\right\rangle$ be the Galois group of $K / \mathbb{Q}$ and $c l_{K}$ the group of ideal classes in $K$. Then there is a $\mathbb{Z}_{l} G$-module isomorphism

$$
\mathbb{Z}_{l} \otimes_{\mathbb{Z}} c l_{K} \simeq \bigoplus_{i=1}^{r-1} \mathbb{Z}_{l} G /\left\langle 1+g_{0}+\ldots+g_{0}^{l-1},\left(g_{0}-1\right)^{h_{i}}\right\rangle
$$

with unique natural numbers $h_{i}$ (see the proof of Lemma 2.1). We fix classes $\mathfrak{C}_{i}$ in $c l_{K}, 1 \leq i \leq r-1$, of order a power of $l$, so that the image of $\mathfrak{C}_{i}$ in $\mathbb{Z}_{l} \otimes c l_{K}$ generates the $i$ th component under this isomorphism. Let $\mathfrak{p}_{j}$ be the prime of $K$ above $p_{j}$ and write

$$
\left[\mathfrak{p}_{j}\right]=\prod_{i=1}^{r-1} \mathfrak{C}_{i}^{b_{i j}\left(g_{0}-1\right)^{h_{i}-1}}, \quad 1 \leq j \leq r,
$$

in $\mathbb{Z}_{l} \otimes c l_{K}$. Define $B_{k}$ to be $(-1)^{k+1}$ times the determinant of the matrix $\left(b_{i j}\right)$ with the $k$ th column deleted $(1 \leq k \leq r)\left({ }^{2}\right)$. These elements $B_{k}$ serve

1991 Mathematics Subject Classification: Primary 11R33; Secondary 11R29, 11R37.

We acknowledge financial support provided by the DFG and NSERC.

$\left({ }^{1}\right)$ At the same time, it fits into a more general setting (see $[\mathrm{Bu}]$ ).

$\left({ }^{2}\right)$ If $r=1$, we must set $B_{1}=1$. 
as a bridge between $c l_{K}$ and the cyclotomic unit group $\mathcal{E}_{K}$ in the unit group $E_{K}$ of $K$. Indeed, set

$$
\xi_{K}=\prod_{1 \neq d \mid n} N_{\mathbb{Q}\left(\zeta_{n}\right) / K}\left(1-\zeta_{n}^{n / d}\right) .
$$

Then

$$
\xi_{K}^{g_{0}-1}=\alpha_{\infty}^{\left(g_{0}-1\right)^{h+1}}
$$

with a unique $\alpha_{\infty} \in K$ (up to rational factors) such that the norm $N_{K / \mathbb{Q}}\left(\alpha_{\infty}\right)$ of $\alpha_{\infty}$ is not an $l$ th power. It is readily seen that $B_{j}$ and the $\mathfrak{p}_{j}$-value $v_{\mathfrak{p}_{j}}\left(\alpha_{\infty}\right)$ of $\alpha_{\infty}$ are proportional modulo $l$, independent of $j$. The Lifted Root Number Conjecture now predicts this ratio to equal a certain number $c$ defined in Lemma 2.3 (and is, in this case, equivalent to that equality). In Section 2 we also review the classical material that has been referred to here. To get a clear picture of what is going on, note that the above $h$ is such that $\left|\mathbb{Z}_{l} \otimes c l_{K}\right|=l^{h}$ and that $\xi_{K}^{g_{0}-1}$ generates $\mathbb{Z}_{l} \otimes \mathcal{E}_{K}$ in $\mathbb{Z}_{l} \otimes E_{K}=\left\langle\alpha_{\infty}^{g_{0}-1}\right\rangle$.

The actual connection to the Lifted Root Number Conjecture is explained in Section 3. However, it would go beyond the scope of this paper to go into detail here, so the reader is referred to [GRW1] $\left({ }^{3}\right)$. In this section we characterize certain maps $\Delta S^{\prime} \stackrel{\varphi}{\rightarrow} E_{S^{\prime}}$ which when injective have cohomologically trivial cokernel. Here, $K / \mathbb{Q}$ may be replaced by an arbitrary cyclic extension $K / k$ of number fields, and $S^{\prime}$ is a finite, sufficiently large $\operatorname{Gal}(K / k)$-set of primes of $K, \Delta S^{\prime}$ the augmentation submodule in the free $G$-module $\mathbb{Z} S^{\prime}$ on the $\mathbb{Z}$-basis $\mathfrak{p} \in S^{\prime}$, and finally $E_{S^{\prime}}$ the group of $S^{\prime}$-units in $K$. We close Section 3 by restating the Lifted Root Number Conjecture in terms of the cokernel of an injective $\varphi$.

The next section recalls the notion of a Ramachandra map $\varphi_{\infty}: \Delta S_{\infty} \rightarrow$ $E_{K}$, where $S_{\infty}$ is the set of all infinite primes of $K$. The Ramachandra $\varphi_{\infty}$ has been used in [RW] to prove the so-called Strong Stark Conjecture for absolutely abelian $K$ (in which 2 is unramified). Here we now extend it to a $\varphi$ as in Section 3 and show that, for our purposes, it suffices to work with a $G$-set $S$ of primes which is large in the restricted sense that the order of the $S$-class group of $K$ is prime to $|G|$.

Section 5 then gives the construction of an isomorphism $\varphi: \mathbb{Z}_{l} \otimes \Delta S \rightarrow$ $\mathbb{Z}_{l} \otimes E_{S}$ in our example. This $\varphi$ extends $\varphi_{\infty}$, with $S$ the set generated by $\left\{\infty, \mathfrak{p}_{1}, \ldots, \mathfrak{p}_{r}, \mathfrak{q}_{1}, \ldots, \mathfrak{q}_{r-1}, \mathfrak{q}_{0}\right\}$, where $\infty$ is a fixed infinite prime, $\mathfrak{q}_{i} \in$ $\mathfrak{C}_{i}(1 \leq i \leq r-1)$ and $\mathfrak{q}_{0}$ a suitably chosen prime which is inert over $\mathbb{Q}$. The Lifted Root Number Conjecture amounts to certain $l$-adic congruences between the Tate-Stark numbers $A_{\varphi}(\chi)$, where $\chi$ runs through the characters of $G$.

$\left({ }^{3}\right)$ In fact, only the second part of the proof of Proposition 3.2 requires more than is in [GRW2]. 
We need to insert a short section, $\S 6$, on Euler systems before we can complete the calculation in a restricted situation.

TheOREM. The Lifted Root Number Conjecture holds true for $K / \mathbb{Q}$ when $r \leq 2$.

Section 6 recalls some basic facts regarding the Euler system

$$
Q \mapsto \xi_{Q}=N_{\mathbb{Q}\left(\zeta_{n}, \zeta_{Q}\right) / K\left(\zeta_{Q}\right)}\left(1-\zeta_{n} \zeta_{Q}\right)
$$

with $Q$ running through the squarefree products of rational primes $q$ splitting in $K$. We employ it, in Section 7 , to get a prime $\mathfrak{q}_{1} \in \mathfrak{C}_{1}$ for which the corresponding Kolyvagin number $\kappa_{q_{1}}$ provides congruences modulo $\mathfrak{p}_{j}(j=$ $1,2)$ that lead to the proof of the theorem.

In the case $r=2$ we can arrange that $\kappa_{q_{1}}$ has norm 1 and so a $\left(g_{0}-1\right)$ th root of it is an $\alpha_{\mathfrak{q}_{1}}$ in the sense of Section 5. By means of local symbols we relate the $\mathfrak{p}_{j}$-value of $\alpha_{\mathfrak{q}_{1}}$ and the congruence class $\bmod \mathfrak{p}_{j}$ of $\kappa_{\mathfrak{q}_{1}}$. For $r \geq 3$ it seems necessary to take repeated $\left(g_{0}-1\right)$ th roots and so such congruences on $\kappa$-values would not be decisive.

2. Conjecture (C). We maintain the notation of the introduction and set

- $\widehat{G}=\widehat{g}_{0}=\sum_{\nu=0}^{l-1} g_{0}^{\nu}$,

- $P_{K}=$ group of principal ideals of $K$,

- $I_{K}=$ group of all ideals of $K$,

and correspondingly with $K$ replaced by $\mathbb{Q}$.

LEMma $2.1\left(^{4}\right)$. (a) $\mathbb{Z}_{l} \otimes E_{K} \simeq \mathbb{Z}_{l} G / \widehat{G}$.

(b) The $\mathfrak{p}_{j}, 1 \leq j \leq r$, constitute an $\mathbb{F}_{l}$-basis of $I_{K}^{G} / I_{\mathbb{Q}}$.

(c) $P_{K}^{G} / P_{\mathbb{Q}}$ has order $l$.

(d) (1.1) holds.

For the proof observe that $\mathbb{Z}_{l} G / \widehat{G} \simeq \mathbb{Z}_{l}\left[\zeta_{l}\right]$ is a discrete valuation ring with prime element the image of $g_{0}-1$. As $l \neq 2, K$ is totally real and $\widehat{G}$ annihilates $\mathbb{Z}_{l} \otimes E_{K}$. Thus (a) is a consequence of Dirichlet's unit theorem. Therefore $H^{1}\left(G, E_{K}\right)=\mathbb{F}_{l}$ and $H^{2}\left(G, E_{K}\right)=0$. Thus, from $E_{K} \longmapsto K^{\times} \rightarrow$ $P_{K}$, we see that $H^{1}\left(G, P_{K}\right)=0$ and $P_{K}^{G} / P_{\mathbb{Q}}$ has order $l$, proving (c); (b) is obvious. For (d) use $P_{K} \longmapsto I_{K} \rightarrow c l_{K}$ in order to arrive at $P_{K}^{G} / P_{\mathbb{Q}} \longmapsto$ $I_{K}^{G} / I_{\mathbb{Q}} \rightarrow c l_{K}^{G}$, whence $c l_{K}^{G} \simeq \mathbb{F}_{l}^{r-1}$ by (b) and (c). Since $\widehat{G}$ annihilates $c l_{K}$ there exist unique numbers $s$ and $h_{1} \geq \ldots \geq h_{s} \geq 1$ such that $\mathbb{Z}_{l} \otimes c l_{K} \simeq$ $\bigoplus_{i=1}^{s} \mathbb{Z}_{l} G /\left\langle\widehat{G},\left(g_{0}-1\right)^{h_{i}}\right\rangle$. Taking fixed points shows $s=r-1$.

$\left({ }^{4}\right)$ The lemma collects well-known facts (see e.g. [Cc] or [La, XIII,4]), which also follow from the theory of genus fields $[\mathrm{Fr}]$. 
Lemma $2.2\left(^{5}\right)$. There exists $h \geq 0$ so that $\xi_{K}^{g_{0}-1}=\alpha_{\infty}^{\left(g_{0}-1\right)^{h+1}}$ with $\alpha_{\infty} \in K^{\times}$satisfying:

(i) $\alpha_{\infty}^{\widehat{G}} \notin \mathbb{Q}^{\times^{l}}$.

(ii) $\operatorname{supp}\left(\alpha_{\infty}\right) \subset\left\{\mathfrak{p}_{1}, \ldots, \mathfrak{p}_{r}\right\}$ and $\left(\alpha_{\infty}\right)$ generates $P_{K}^{G} / P_{\mathbb{Q}}$.

(iii) $\alpha_{\infty}$ is unique up to rational factors.

Above, $\operatorname{supp}\left(\alpha_{\infty}\right)$ is the set of prime divisors of the principal ideal $\left(\alpha_{\infty}\right)$ generated by $\alpha_{\infty}$.

The proof of the lemma is based on the fact that $\xi_{K}^{g_{0}-1} \neq 1$, which is due to Ramachandra [Wa, Theorem 8.3]. Since $\xi_{K}^{g_{0}-1} \in E_{K}$, there is, by Lemma 2.1(a), a maximal $h \geq 0$ with $\xi_{K}^{g_{0}-1}=v^{\left(g_{0}-1\right)^{h}}$ and $v \in E_{K}$. As $\xi_{K}^{g_{0}-1}$ has norm 1 and $l$ is odd, we may assume that $v$ has norm 1 . Hence there exists $\alpha_{\infty} \in K^{\times}$with $\alpha_{\infty}^{g_{0}-1}=v$. In particular $\left(\alpha_{\infty}\right) \in P_{K}^{G}$. Suppose that $\left(\alpha_{\infty}\right)$ is in the image of $P_{\mathbb{Q}}$ in $P_{K}^{G}$, i.e., $\alpha_{\infty}=a \cdot v_{1}$ with $a \in \mathbb{Q}^{\times}$and a unit $v_{1}$. Then $v=v_{1}^{g_{0}-1}$ contradicts the maximality of $h$. Consequently, $\left(\alpha_{\infty}\right)$ generates $P_{K}^{G} / P_{\mathbb{Q}}$ and is a product of the $\mathfrak{p}_{j}$ times a rational number. Modifying $\alpha_{\infty}$ by the inverse of this rational number proves (i) and (ii).

If $h=0$, then (iii) is obvious. If $h>0$, then $\alpha_{1}^{\left(g_{0}-1\right)^{h+1}}=\alpha_{\infty}^{\left(g_{0}-1\right)^{h+1}}$ leads to $\alpha_{1}^{\left(g_{0}-1\right)^{h}}=\alpha_{\infty}^{\left(g_{0}-1\right)^{h}} \cdot a$ for some $a \in \mathbb{Q}^{\times}$, and taking norms yields $a^{l}=1$, hence $a=1$. This argument can be repeated.

As in the introduction (see $(1.2))$, we write $\left[\mathfrak{p}_{j}\right]=\prod_{i=1}^{r-1} \mathfrak{C}_{i}^{b_{i j}\left(g_{0}-1\right)^{h_{i}-1}}$ in $\mathbb{Z}_{l} \otimes c l_{K}$ with integers $b_{i j}$. The proof of Lemma 2.1 shows that the matrix $\left(b_{i j}\right)_{\substack{1 \leq i \leq r-1 \\ 1 \leq j \leq r}}$ has rank $r-1$ over $\mathbb{F}_{l}$, whence the row vector $B_{k}=$ $(-1)^{k+1} \operatorname{det}\left(b_{i j}\right)$ is non-zero modulo $l\left({ }^{6}\right)$. It satisfies $\left(b_{i j}\right) \cdot\left(B_{1}, \ldots, B_{r}\right)^{T}=$ $(0, \ldots, 0)$. Since, by $(1.2),\left(b_{i j}\right)$ is the matrix of the $\mathbb{F}_{l^{-}}$-linear map $I_{K}^{G} / I_{\mathbb{Q}} \rightarrow$ $c l_{K}^{G}$, the ideal $\mathfrak{p}_{1}^{B_{1}} \ldots \mathfrak{p}_{r}^{B_{r}}$ is principal. By Lemma 2.2(ii) we see that there is a $\widetilde{c} \in \mathbb{Z}, \widetilde{c} \not \equiv 0 \bmod l$ such that

$$
-v_{\mathfrak{p}_{j}}\left(\alpha_{\infty}\right) \equiv \widetilde{c} \cdot B_{j} \bmod l
$$

for $1 \leq j \leq r$, where $v_{\mathfrak{p}}(x)$, for a prime ideal $\mathfrak{p}$ of $K$ and an $x \in K$, denotes the $\mathfrak{p}$-value of $x$.

LEMMA 2.3. Let $\mathfrak{q}_{i} \in \mathfrak{C}_{i}, 1 \leq i \leq r-1$, be primes of prime absolute norm $q_{i}$ which are different from $p_{1}, \ldots, p_{r}$. Furthermore, let $q_{0}$ be a rational prime with Artin symbol $\left(q_{0}, K / \mathbb{Q}\right)=g_{0}$. Form the matrix $\left(c_{i j}\right)$ by means of the local norm residue symbols

$\left({ }^{5}\right)$ Compare (1.3) in Section 1.

$\left({ }^{6}\right)$ Recall that $B_{1}=1$ if $r=1$. 


$$
\left(q_{i}, K_{\mathfrak{p}_{j}} / \mathbb{Q}_{p_{j}}\right)=g_{0}^{c_{i j}}, \quad 0 \leq i \leq r-1,1 \leq j \leq r\left({ }^{7}\right) .
$$

Define $c=\operatorname{det} c_{i j}$. Then $c \not \equiv 0 \bmod l$.

Note that due to Chebotarev's density theorem such prime ideals $\mathfrak{q}_{i}$ exist. Note also that the $c_{i j}$, and equally well the $b_{i j}$ and the $B_{j}$, depend on the choice of the $\mathfrak{C}_{i}$.

Before turning to the proof of Lemma 2.3 we specify the conjecture that has been indicated in the introduction:

$$
\widetilde{c} \equiv c \bmod l .
$$

Proof (of Lemma 2.3). Define $\widetilde{K}$ to be the Hilbert $l$-class field of $K$ and let $\widehat{K} / \mathbb{Q}$ be the maximal abelian subextension of $\widetilde{K} / \mathbb{Q}\left(^{8}\right)$. The Artin symbol $(, \widetilde{K} / K): \mathbb{Z}_{l} \otimes c l_{K} \rightarrow \operatorname{Gal}(\widetilde{K} / K)$ is an isomorphism and so provides the exact sequence $\mathbb{Z}_{l} \otimes c l_{K} \longmapsto \operatorname{Gal}(\widetilde{K} / \mathbb{Q}) \rightarrow G$. Since $G$ is cyclic, it follows that also $\mathbb{Z}_{l} \otimes c l_{K} / c l_{K}^{g_{0}-1} \longmapsto \operatorname{Gal}(\widehat{K} / \mathbb{Q}) \rightarrow G$ is exact, whence $(, \widetilde{K} / K)$ : $\mathbb{Z}_{l} \otimes c l_{K} / c l_{K}^{g_{0}-1} \stackrel{\sim}{\rightarrow} \operatorname{Gal}(\widehat{K} / K)$.

Set $\sigma_{i}=\left(q_{i}, \widehat{K} / \mathbb{Q}\right), 0 \leq i \leq r-1$. The choice of the $\mathfrak{q}_{i}$ guarantees that $\sigma_{i}=\left(\mathfrak{q}_{i}, \widehat{K} / K\right), 1 \leq i \leq r-1$, is an $\mathbb{F}_{l}$-basis of $\operatorname{Gal}(\widehat{K} / K)$. Observe here that $l$ annihilates $\mathbb{Z}_{l} \otimes c l_{K} / c l_{K}^{g_{0}-1}$ because the ideals $\left\langle g_{0}-1, \widehat{G}\right\rangle$ and $\left\langle g_{0}-1, l\right\rangle$ coincide. In particular, $\operatorname{Gal}(\widehat{K} / \mathbb{Q})$ has order $l^{r}$. Since $\widehat{K}$ contains the composite of the subextensions of degree $l$ of all $\mathbb{Q}\left(\zeta_{p_{j}}\right), 1 \leq j \leq r$, it therefore coincides with it and $\operatorname{Gal}(\widehat{K} / \mathbb{Q})$ is $l$-elementary. As a consequence, $\sigma_{0}, \sigma_{1}, \ldots, \sigma_{r-1}$ is an $\mathbb{F}_{l}$-basis of $\operatorname{Gal}(\widehat{K} / \mathbb{Q})$ because $\sigma_{0}$ restricts to $g_{0}$ on $K$, and the map

$$
\prod_{j=1}^{r} U_{p_{j}} / U_{p_{j}}^{l} \rightarrow \operatorname{Gal}(\widehat{K} / \mathbb{Q}), \quad\left(u_{j}\right) \mapsto \prod_{j=1}^{r}\left(u_{j}, \widehat{K}_{\widehat{\mathfrak{p}}_{j}} / \mathbb{Q}_{p_{j}}\right)
$$

is an isomorphism. Here, $U_{p_{j}}$ is the unit group in $\mathbb{Q}_{p_{j}}$ and $\widehat{\mathfrak{p}}_{j}$ a prime of $\widehat{K}$ above $\mathfrak{p}_{j}$. Note that the 1 -units in $\mathbb{Q}_{p_{j}}$ are all $l$ th powers, so $\left[U_{p_{j}}: U_{p_{j}}^{l}\right]=l$.

The isomorphism takes $q_{i}$, viewed in $\prod_{j=1}^{r} U_{p_{j}} / U_{p_{j}}^{l}$ on the diagonal, to

$$
\prod_{j=1}^{r}\left(q_{i}, \widehat{K}_{\widehat{\mathfrak{p}}_{j}} / \mathbb{Q}_{p_{j}}\right)=\left(q_{i}, \widehat{K}_{\widehat{\mathfrak{q}}_{i}} / \mathbb{Q}_{q_{i}}\right)^{-1}=\sigma_{i}^{-1}, \quad 0 \leq i \leq r-1,
$$

by reciprocity. This shows that $q_{0}, \ldots, q_{r-1}$ is an $\mathbb{F}_{l}$-basis of $\prod_{j=1}^{r} U_{p_{j}} / U_{p_{j}}^{l}$.

$\left({ }^{7}\right)$ For a number field $L$ and a prime $\mathfrak{p}$ of $L, L_{\mathfrak{p}}$ denotes the completion of $L$ at $\mathfrak{p}$.

$\left.{ }^{8}\right) \widehat{K}$ is the genus field of $K / \mathbb{Q}$. 
Since $\prod_{j=1}^{r}\left(, K_{\mathfrak{p}_{j}} / \mathbb{Q}_{p_{j}}\right): \prod_{j=1}^{r} U_{p_{j}} / U_{p_{j}}^{l} \rightarrow G^{r}$ is an isomorphism, the standard basis of $G^{r}$ is the image of certain $\prod_{s=0}^{r-1} q_{s}^{x_{i s}}$, i.e.,

$$
\left(\prod_{s=0}^{r-1} q_{s}^{x_{i s}}, K_{\mathfrak{p}_{j}} / \mathbb{Q}_{p_{j}}\right)= \begin{cases}g_{0} & \text { if } i=j \\ 1 & \text { if } i \neq j .\end{cases}
$$

This implies

$$
\prod_{s=0}^{r-1}\left(q_{s}, K_{\mathfrak{p}_{j}} / \mathbb{Q}_{p_{j}}\right)^{x_{i s}}=g_{0}^{\sum_{s=0}^{r-1} x_{i s} c_{s j}}=g_{0}^{\delta_{i j}}
$$

and finishes the proof $\left({ }^{9}\right)$.

3. The Lifted Root Number Conjecture for $K$. In this section $K / k$ is a cyclic extension of number fields with group $G=\left\langle g_{0}\right\rangle$ where $g_{0}$ is the Frobenius automorphism of some fixed prime $\mathfrak{q}_{0}$ of $K$ which is inert over $k$. We let $S^{\prime}$ denote a finite $G$-set of primes of $K$ containing $\mathfrak{q}_{0}$, all infinite primes, all ramified primes for the extension $K / k$, and enough primes to generate the class group $c l_{K}$. Our aim is to characterize certain maps $\Delta S^{\prime} \stackrel{\varphi}{\rightarrow} E_{S^{\prime}}$, which whenever injective have cohomologically trivial cokernel, and to restate the Lifted Root Number Conjecture in terms of them.

Lemma 3.1. Let $\mathfrak{p}$ be a prime of $K, g_{\mathfrak{p}}$ a generator of its decomposition group $G_{\mathfrak{p}}$ (with respect to $k$ ) and $a_{\mathfrak{p}} \in k_{\mathfrak{p}}^{\times}$so that $\left(a_{\mathfrak{p}}, K_{\mathfrak{p}} / k_{\mathfrak{p}}\right)=g_{\mathfrak{p}}$ (with $k_{\mathfrak{p}}$ denoting the completion of $k$ in $\left.K_{\mathfrak{p}}\right)$. Then the extension class of the bottom row sequence in the push-out diagram

$$
\begin{array}{rlcccc}
\mathbb{Z} & \stackrel{\widehat{G}_{\mathfrak{p}}}{\longmapsto} & \mathbb{Z} G_{\mathfrak{p}} & g_{\mathfrak{p}}-1 & & \\
\downarrow & & \downarrow & & \Delta G_{\mathfrak{p}} \\
\downarrow & & \|
\end{array}, \quad \text { with respect to the map } 1 \mapsto a_{\mathfrak{p}},
$$

corresponds to the local fundamental class of $K_{\mathfrak{p}} / k_{\mathfrak{p}}$ under the canonical isomorphisms

$$
\operatorname{Ext}_{G_{\mathfrak{p}}}^{1}\left(\Delta G_{\mathfrak{p}}, K_{\mathfrak{p}}^{\times}\right) \simeq H^{1}\left(G_{\mathfrak{p}}, \operatorname{Hom}\left(\Delta G_{\mathfrak{p}}, K_{\mathfrak{p}}^{\times}\right)\right) \simeq H^{2}\left(G_{\mathfrak{p}}, K_{\mathfrak{p}}^{\times}\right) .
$$

For a proof see [Sn, pp. 52-53].

The exact sequence $\mathbb{Z} \stackrel{\widehat{G}}{\longmapsto} \mathbb{Z} G \stackrel{g_{0}-1}{\rightarrow} \Delta G$ tensored with $\Delta S^{\prime}$ yields the new exact sequence

$$
\Delta S^{\prime} \longmapsto \Delta S^{\prime} \otimes \mathbb{Z} G \rightarrow \Delta S^{\prime} \otimes \Delta G .
$$

Let $S_{*}^{\prime}$ be a set of $G$-representatives for $S^{\prime}$ and set $g_{\mathfrak{p}}=g_{0}^{\left[G: G_{\mathfrak{p}}\right]}$ for $\mathfrak{p} \in S_{*}^{\prime}$, so $\left\langle g_{\mathfrak{p}}\right\rangle=G_{\mathfrak{p}}$.

$\left({ }^{9}\right) \delta_{i j}$ is the Kronecker symbol. 
Proposition 3.2. Assume that for each $\mathfrak{p} \in S_{*}^{\prime}, \mathfrak{p} \neq \mathfrak{q}_{0}$, we are given an element $\alpha_{\mathfrak{p}} \in K^{G_{\mathfrak{p}}} \cap E_{S^{\prime}}$ satisfying

$$
\left(\alpha_{\mathfrak{p}}, K_{\mathfrak{p}^{\prime}} /\left(K^{G_{\mathfrak{p}}}\right)_{\mathfrak{p}^{\prime}}\right)= \begin{cases}g_{\mathfrak{p}} & \text { for } \mathfrak{p}^{\prime}=\mathfrak{p}, \\ 1 & \text { for } \mathfrak{p}^{\prime} \neq \mathfrak{p}, \mathfrak{q}_{0},\end{cases}
$$

where $\mathfrak{p}^{\prime}$ runs through the primes of $K$. Then the $G$-map $\varphi: \Delta S^{\prime} \rightarrow E_{S^{\prime}}$ defined by $\mathfrak{p}-\mathfrak{q}_{0} \mapsto \alpha_{\mathfrak{p}}$ for $\mathfrak{p} \in S_{*}^{\prime}, \mathfrak{p} \neq \mathfrak{q}_{0}$, takes the extension class in $\operatorname{Ext}_{G}^{1}\left(\Delta S^{\prime} \otimes \Delta G, \Delta S^{\prime}\right)$ of $(\Delta)$ to the Tate class $\tau_{S^{\prime}} \in \operatorname{Ext}_{G}^{1}\left(\Delta S^{\prime} \otimes \Delta G, E_{S^{\prime}}\right)$.

REMARK. More precisely, tensoring the augmentation sequence $\Delta G \longmapsto$ $\mathbb{Z} G \rightarrow \mathbb{Z}$ with $\Delta S^{\prime}$ induces an isomorphism

$$
\operatorname{Ext}_{G}^{1}\left(\Delta S^{\prime} \otimes \Delta G, E_{S^{\prime}}\right) \rightarrow \operatorname{Ext}_{G}^{2}\left(\Delta S^{\prime}, E_{S^{\prime}}\right)
$$

sending $\tau_{S^{\prime}}$ to what is usually regarded as the Tate class [GRW1].

Proof (of Proposition 3.2). We begin by picking for each $\mathfrak{p} \in S_{*}^{\prime}$ an element $a_{\mathfrak{p}}$ in $\left(K^{G_{\mathfrak{p}}}\right)_{\mathfrak{p}}$ so that $\left(a_{\mathfrak{p}}, K_{\mathfrak{p}} /\left(K^{G_{\mathfrak{p}}}\right)_{\mathfrak{p}}\right)=g_{\mathfrak{p}}$. To $a_{\mathfrak{p}}$ we then assign the idèle $a_{(\mathfrak{p})}$ in the $S^{\prime}$-idèle group $J_{K^{G_{\mathfrak{p}}, S^{\prime}}}$ of $K^{G_{\mathfrak{p}}}$, which has component 1 everywhere except at the prime $\mathfrak{p} \cap K^{G_{\mathfrak{p}}}$ where the component shall be $a_{\mathfrak{p}}$. The element $\alpha_{\mathfrak{p}}$ viewed as principal idèle will be denoted by $\alpha_{(\mathfrak{p})}$.

We claim:

$$
a_{(\mathfrak{p})} \equiv a_{\left(\mathfrak{q}_{0}\right)} \alpha_{(\mathfrak{p})} \bmod N_{K / K^{G_{\mathfrak{p}}}} J_{K}
$$

This is checked for each prime $\mathfrak{p}^{\prime} \cap K^{G_{\mathfrak{p}}}$ at a time. Note that $\mathfrak{p}$ and $\mathfrak{q}_{0}$ are non-split in $K / K^{G_{\mathfrak{p}}}$.

At $\mathfrak{p}^{\prime} \neq \mathfrak{p}, \mathfrak{q}_{0}$ the two idèles $a_{(\mathfrak{p})}$ and $a_{\left(\mathfrak{q}_{0}\right)}$ are 1 , and $\alpha_{(\mathfrak{p})}$ is a local norm. At $\mathfrak{p}^{\prime}=\mathfrak{p}$ the two idèles $a_{(\mathfrak{p})}$ and $\alpha_{(\mathfrak{p})}$ differ by a local norm and $a_{\left(\mathfrak{q}_{0}\right)}$ is 1 . At $\mathfrak{p}^{\prime}=\mathfrak{q}_{0}$ the reciprocity law implies $\left(\alpha_{\mathfrak{p}}, K_{\mathfrak{q}_{0}} /\left(K^{G_{\mathfrak{p}}}\right)_{\mathfrak{q}_{0}}\right)=g_{\mathfrak{p}}^{-1}, a_{(\mathfrak{p})}$ is 1 , and $\left(a_{\mathfrak{q}_{0}}, K_{\mathfrak{q}_{0}} /\left(K^{G_{\mathfrak{q}_{0}}}\right)_{\mathfrak{q}_{0}}\right)=g_{0}$ becomes $g_{0}^{\left[G: G_{\mathfrak{p}}\right]}=g_{\mathfrak{p}}$ in $\operatorname{Gal}\left(K_{\mathfrak{q}_{0}} /\left(K^{G_{\mathfrak{p}}}\right)_{\mathfrak{q}_{0}}\right)$ as follows from the commutativity of

$$
\begin{array}{ccc}
k_{\mathfrak{q}_{0}}^{\times} & \stackrel{\left(, K_{\mathfrak{q}_{0}} / k_{\mathfrak{q}_{0}}\right)}{\longrightarrow} & \operatorname{Gal}\left(K_{\mathfrak{q}_{0}} / k_{\mathfrak{q}_{0}}\right) \\
\downarrow & & \downarrow\left[G: G_{\mathfrak{p}}\right]=t \\
\left(K^{G_{\mathfrak{p}}}\right)_{\mathfrak{q}_{0}}^{\times} \stackrel{\left(, K_{\mathfrak{q}_{0}} /\left(K^{G} G_{\mathfrak{p}}\right)_{\mathfrak{q}_{0}}\right)}{\longrightarrow} & \operatorname{Gal}\left(K_{\mathfrak{q}_{0}} /\left(K^{G_{\mathfrak{p}}}\right)_{\mathfrak{q}_{0}}\right)
\end{array}
$$

with $t$ denoting the transfer map [Se, VII,8].

Since outside of $S^{\prime}$ the extension $K / k$ is unramified and since local units are norms in local unramified extensions, we will even find $\beta_{(\mathfrak{p})} \in J_{K, S^{\prime}}$ such that

$$
a_{(\mathfrak{p})} N_{K / K^{G_{\mathfrak{p}}}}\left(\beta_{(\mathfrak{p})}\right)=a_{\left(\mathfrak{q}_{0}\right)} \alpha_{(\mathfrak{p})} .
$$

Recall that here $\mathfrak{p} \in S_{*}^{\prime}, \mathfrak{p} \neq \mathfrak{q}_{0}$. We temporarily set $\alpha_{\left(\mathfrak{q}_{0}\right)}=\beta_{\left(\mathfrak{q}_{0}\right)}=1$.

The rest of the proof of the proposition consists of combining these data with the construction of a Tate sequence (see e.g. [We, Chapter 5]). 
For each $\mathfrak{p} \in S_{*}^{\prime}$ we take the diagram of Lemma 3.1 with the middle vertical map denoted by $\mu_{\mathfrak{p}}$. Inducing these up to $G$ and building the direct sum over $S_{*}^{\prime}$ we get

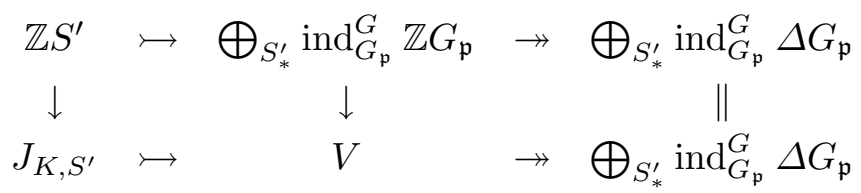

where we have glued on the unit idèles outside $S^{\prime}$ in $J$ and $V$. We modify the left vertical map by sending $\mathfrak{p} \in S_{*}^{\prime}$ to $a_{(\mathfrak{p})} N_{K / K^{G_{\mathfrak{p}}}} \beta_{(\mathfrak{p})}$, and the middle one by sending the free $G$-module generator ind $\left(1_{\mathfrak{p}}\right)$ of $\operatorname{ind}_{G_{\mathfrak{p}}}^{G} \mathbb{Z} G_{\mathfrak{p}}$ to $\mu_{\mathfrak{p}}\left(1_{\mathfrak{p}}\right) \beta_{(\mathfrak{p})}$, where now $\beta_{(\mathfrak{p})} \in J_{K, S^{\prime}}$ is read in $V$. Then the new diagram still commutes. It is the top face in

(D)

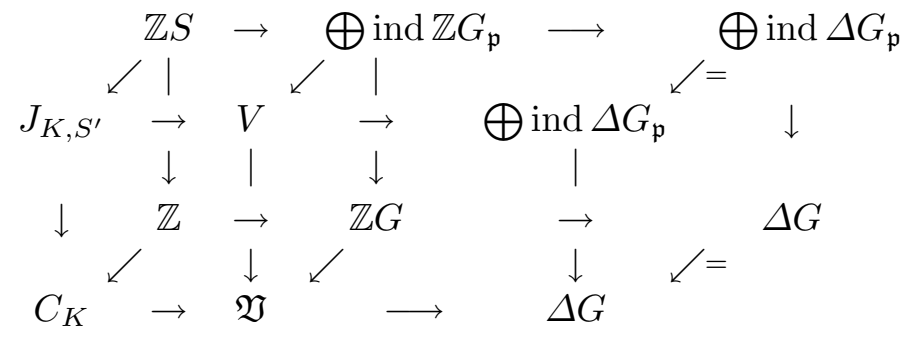

The bottom face of (D) is the diagram of Lemma 3.1 for $\mathfrak{p}=\mathfrak{q}_{0}$ composed with the push-out diagram along the natural map from $K_{\mathfrak{q}_{0}}^{\times}$into the idèle class group $C_{K}$ of $K$ :

$$
\begin{array}{cccccc}
K_{\mathfrak{q}_{0}}^{\times} & \longmapsto & V_{\mathfrak{q}_{0}} & \rightarrow & \Delta G_{\mathfrak{q}_{0}} \\
\downarrow & & \downarrow & & \| \\
C_{K} & \longmapsto & \mathfrak{V} & \rightarrow & \Delta G
\end{array}
$$

Remember that $G_{\mathfrak{q}_{0}}=G$.

By the compatibility of local and global fundamental classes the bottom row has extension class corresponding to the global fundamental class.

The commutative diagram



with middle arrow $x \mapsto x\left(1+g_{0}+\ldots+g_{0}^{\left[G: G_{\mathfrak{p}}\right]-1}\right)$ induces the back face in (D). The right face of (D) clearly commutes. The left face commutes because the idèle class of $a_{(\mathfrak{p})} N_{K / K^{G_{\mathfrak{p}}}} \beta_{(\mathfrak{p})}$, for $\mathfrak{p} \in S_{*}^{\prime}, \mathfrak{p} \neq \mathfrak{q}_{0}$, is the same as that of $a_{\left(q_{0}\right)}$.

On observing that the left half of the top face in (D) is a push-out square for $V$ we obtain a unique map $V \rightarrow \mathfrak{V}$ making the whole diagram commute. 
As $S^{\prime}$ is sufficiently large, $J_{K, S^{\prime}} \rightarrow C_{K}$ is surjective; since $\mathfrak{q}_{0} \in S_{*}^{\prime}$, $\bigoplus_{S_{*}^{\prime}} \operatorname{ind}_{G_{\mathfrak{p}}}^{G} \Delta G_{\mathfrak{p}} \rightarrow \Delta G$ is surjective. The kernels of the vertical arrows in (D) fit into

$$
\begin{array}{rllll}
\Delta S^{\prime} & \longmapsto & B & \rightarrow & L \\
\downarrow \varphi & & \downarrow & & \| \\
E_{S^{\prime}} & \longmapsto & A & \rightarrow & L
\end{array}
$$

and $\varphi$ takes $\mathfrak{p}-\mathfrak{q}_{0}$ to $a_{(\mathfrak{p})} N_{K / K^{G_{\mathfrak{p}}}} \beta_{(\mathfrak{p})} / a_{\left(\mathfrak{q}_{0}\right)}$ which is the principal idèle $\alpha_{(\mathfrak{p})}$.

We now compare this with the kernels of the vertical maps in

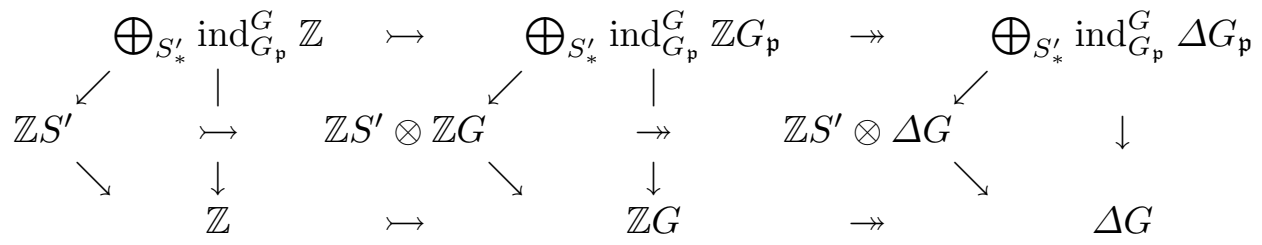

with outer southwest arrows $g \otimes_{G_{\mathfrak{p}}} 1 \mapsto g \mathfrak{p}$ and middle one $g \otimes_{G_{\mathfrak{p}}} x \mapsto$ $g \mathfrak{p} \otimes g x y_{\mathfrak{p}}$ where $y_{\mathfrak{p}}=1+g_{0}+\ldots+g_{0}^{\left[G: G_{\mathfrak{p}}\right]-1}$.

This is

$$
\begin{array}{ccccc}
\Delta S^{\prime} & \longmapsto & B & \rightarrow & L \\
\| & & \downarrow & & \downarrow \\
\Delta S^{\prime} & \longmapsto & \Delta S^{\prime} \otimes \mathbb{Z} G & \rightarrow & \Delta S^{\prime} \otimes \Delta G
\end{array}
$$

and we regard the two vertical isomorphisms as identifications. Then

$$
\begin{array}{rcccc}
\Delta S^{\prime} & \longmapsto & \Delta S^{\prime} \otimes \mathbb{Z} G & \rightarrow & \Delta S^{\prime} \otimes \Delta G \\
\downarrow \varphi & & \downarrow & & \| \\
E_{S^{\prime}} & \longmapsto & A & \rightarrow & \Delta S^{\prime} \otimes \Delta G
\end{array}
$$

and the bottom row is the $\tau_{S^{\prime}}$ of the proposition.

REMARK. 1. There always exist such $\varphi$ which are injective. We omit the proof.

2. If $G_{\mathfrak{p}}=1$, then the only restriction on $\alpha_{\mathfrak{p}}$ is to belong to $E_{S^{\prime}}$.

If the $\varphi$ in Proposition 3.2 is injective, we can build the $\Omega_{\varphi}$ as in [GRW1,2] and express the Lifted Root Number Conjecture in terms of a conjectural representing homomorphism for the finite cohomologically trivial module coker $\varphi$.

This is carried out next. Observe that coker $\varphi$ then coincides with the cokernel of the middle map in diagram (T), in which $\Delta S^{\prime} \otimes \mathbb{Z} G$ and $A$ are cohomologically trivial, so it is so itself as well.

The map $\varphi$ induces $\widetilde{\varphi}: B \stackrel{\beta}{\rightarrow} L \oplus \Delta S^{\prime} \stackrel{1 \oplus \varphi}{\longrightarrow} L \oplus E_{S^{\prime}} \stackrel{\alpha}{\rightarrow} A$. Now $B$ and $L$ are just abbreviations for $\Delta S^{\prime} \otimes \mathbb{Z} G$ and $\Delta S^{\prime} \otimes \Delta G$. The auxiliary maps $\beta$ 
and $\alpha$ can be any maps resulting from commuting diagrams

$$
\begin{aligned}
& L \longmapsto B \quad \rightarrow \Delta S^{\prime} \quad E_{S^{\prime}} \longmapsto L \oplus E_{S^{\prime}} \rightarrow L \\
& \downarrow|G| \quad \downarrow \beta \quad\|\quad\| \quad \downarrow \alpha \quad \downarrow d G \mid \\
& L \longmapsto L \oplus \Delta S^{\prime} \rightarrow \Delta S^{\prime} \quad E_{S^{\prime}} \longmapsto \quad A \quad \rightarrow L
\end{aligned}
$$

$\Omega_{\varphi}$ is defined as the element $[\operatorname{coker} \widetilde{\varphi}]-2 \partial(L,|G|)$ in the Grothendieck group $K_{0} T(\mathbb{Z} G)$ of finite cohomologically trivial $\mathbb{Z} G$-modules (see [GRW1 or GRW2]).

Analogously we obtain a map $\widetilde{1}: B \stackrel{\beta_{1}}{\longrightarrow} L \oplus \Delta S^{\prime} \stackrel{1 \oplus 1}{\longrightarrow} L \oplus \Delta S \stackrel{\alpha_{1}}{\rightarrow} B$ and define

$$
\mho_{1}=[\operatorname{coker} \tilde{1}]-2 \partial(L,|G|),
$$

i.e., we have replaced $\varphi: \Delta S^{\prime} \rightarrow E_{S^{\prime}}$ by the identity map $1: \Delta S^{\prime}=\Delta S^{\prime}$ and the Tate sequence $E_{S^{\prime}} \longmapsto A \rightarrow B \rightarrow \Delta S^{\prime}$ by $\Delta S^{\prime} \longmapsto \Delta S^{\prime} \otimes \mathbb{Z} G \rightarrow$ $\Delta S^{\prime} \otimes \mathbb{Z} G \rightarrow \Delta S^{\prime}$, which, as before, is $\mathbb{Z} \stackrel{\widehat{G}}{\longmapsto} \mathbb{Z} G \stackrel{g_{0}-1}{\longrightarrow} \mathbb{Z} G \rightarrow \mathbb{Z}$ tensored with $\Delta S^{\prime}$.

LeMmA 3.3. $\Omega_{\varphi}-\mho_{1}=[\operatorname{coker} \varphi]$.

This follows from the commutativity of a diagram

$$
\begin{array}{ccccccc}
B & \stackrel{\beta_{1}}{\rightarrow} & L \oplus \Delta S^{\prime} & \stackrel{1 \oplus 1}{\longrightarrow} & L \oplus \Delta S^{\prime} & \stackrel{\alpha_{1}}{\rightarrow} & B \\
\| & & \| & & \downarrow 1 \oplus \varphi & & \downarrow \varphi_{0} \\
B & \stackrel{\beta}{\rightarrow} & L \oplus \Delta S^{\prime} & \stackrel{1 \oplus \varphi}{\longrightarrow} & L \oplus E_{S^{\prime}} & \stackrel{\alpha}{\longrightarrow} & A
\end{array}
$$

with suitably chosen $\beta_{1}, \alpha_{1}, \beta, \alpha$, and in which $\varphi_{0}$ is the middle map of diagram $(\mathrm{T})$. For it implies

$$
\begin{array}{ccccc}
B & \stackrel{\widetilde{1}}{\longmapsto} & B & \rightarrow & \text { coker } \widetilde{1} \\
\| & & I_{\varphi_{0}} & & \downarrow \\
B & \stackrel{\widetilde{\varphi}}{\longmapsto} A & A & \rightarrow & \operatorname{coker} \widetilde{\varphi}
\end{array}
$$

so the snake lemma proves the assertion because coker $\varphi_{0}=\operatorname{coker} \varphi$.

In order to see the above claimed commutativity we now define particular maps $\beta=\beta_{1}: B \rightarrow L \oplus \Delta S^{\prime}, \alpha: L \oplus E_{S^{\prime}} \rightarrow A$ and $\alpha_{1}: L \oplus \Delta S^{\prime} \rightarrow B$. To this end, we label, as shown, our maps in the diagrams

$$
\begin{array}{rllllllllllll}
\Delta S^{\prime} & \stackrel{\mu_{1}}{\longrightarrow} & B & \stackrel{\mu_{2}}{\rightarrow} & L & \Delta S^{\prime} & \stackrel{\mu_{1}}{\longmapsto} & B & \stackrel{\mu_{2}}{\rightarrow} & L \\
\downarrow \varphi & & \downarrow \varphi_{0} & \| & \downarrow 1 & & \downarrow & & \| \\
E_{S^{\prime}} & \stackrel{\mu_{3}}{\longmapsto} & A & \stackrel{\mu_{4}}{\longrightarrow} & L & \Delta S^{\prime} & \stackrel{\mu_{1}}{\longmapsto} & B & \stackrel{\mu_{2}}{\rightarrow} & L
\end{array}
$$

and in the right end of the Tate sequence $L \stackrel{\rho_{1}}{\longmapsto} B \stackrel{\rho_{2}}{\rightarrow} \Delta S^{\prime}$.

Choose $\mathbb{Z}$-maps $\mu_{2}^{\prime}, \rho_{1}^{\prime}$ with $\mu_{2} \mu_{2}^{\prime}=\operatorname{id}_{L}=\rho_{1}^{\prime} \rho_{1}$ and build the $G$-maps $\widetilde{\mu}_{2}=\widehat{G} \mu_{2}^{\prime}, \widetilde{\rho}_{1}=\widehat{G} \rho_{1}^{\prime}$. 
The left diagram then gives $\mu_{4} \varphi_{0} \mu_{2}^{\prime}=\mathrm{id}_{L}$. We set $\widetilde{\mu}_{4}=\varphi_{0} \widetilde{\mu}_{2}$ and $\beta(b)=\left(\widetilde{\rho}_{1}(b), \rho_{2}(b)\right), \quad \alpha(y, e)=\widetilde{\mu}_{4}(y)+\mu_{3}(e), \quad \alpha_{1}(y, d)=\widetilde{\mu}_{2}(y)+\mu_{1}(d)$ for $b \in B, y \in L, e \in E_{S^{\prime}}, d \in \Delta S^{\prime}$. Then

$$
\varphi_{0} \alpha_{1}(y, d)=\varphi_{0} \widetilde{\mu}_{2}(y)+\varphi_{0} \mu_{1}(d)=\widetilde{\mu}_{4}(y)+\mu_{3} \varphi(d)=\alpha(1 \oplus \varphi)(y, d) .
$$

Passing to the Hom description of $K_{0} T(\mathbb{Z} G)$ (see Appendix A in [GRW1]), we now have

LEMMA 3.4. $\mho_{1}$ is represented by

$$
a_{S^{\prime}}(\chi)=|G|^{(\chi, \theta)} \prod_{\psi \neq 1}\left(\psi\left(g_{0}\right)-1\right)^{-\left(\chi \psi^{-1}, \theta\right)}\left({ }^{10}\right)
$$

with $\psi$ running through the irreducible characters of $G$ and $\theta$ denoting the character of $\Delta S^{\prime}$.

The proof starts out from the two diagrams

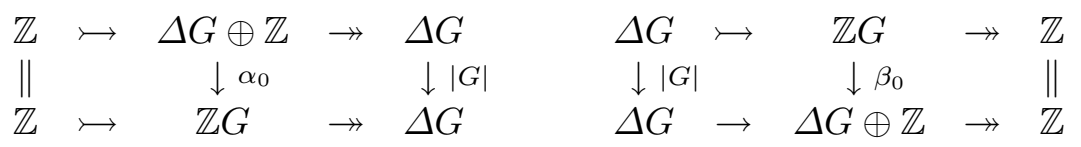

in which

$$
\alpha_{0}(d, z)=d \sum_{i=1}^{|G|-1} i g_{0}^{i}+z \widehat{G} \quad(d \in \Delta G, z \in \mathbb{Z}) \quad \text { and } \quad \beta_{0}(1)=(|G|-\widehat{G}, 1) .
$$

The identity

$$
\left(g_{0}-1\right) \sum_{i=1}^{|G|-1} i g_{0}^{i}=|G|-\widehat{G}
$$

shows the commutativity.

Now,

$$
\left(\alpha_{0} \beta_{0}\right)(1)=(|G|-\widehat{G}) \sum_{i=1}^{|G|-1} i g_{0}^{i}+\widehat{G}=x, \quad \text { say. }
$$

Before proceeding, we note that

$$
\psi\left(\frac{x}{|G|^{2}}\right)= \begin{cases}1 /|G|, & \psi=1 \\ 1 /\left(\psi\left(g_{0}\right)-1\right), & \psi \neq 1\end{cases}
$$

The following computations (including notation) are based on Appendix A in [GRW1]. Tensor the diagrams with $\Delta S^{\prime}$. Then we have

$$
\begin{aligned}
\mho_{1} & =\left[\Delta S^{\prime} \otimes \mathbb{Z} G / \Delta S^{\prime} \otimes \mathbb{Z} G \cdot x\right]-2 \partial\left(\Delta S^{\prime} \otimes \Delta G,|G|\right) \\
& =\partial\left(\Delta S^{\prime} \otimes \mathbb{Z} G, x\right)-2 \partial\left(\Delta S^{\prime} \otimes \mathbb{Z} G,|G|\right)+2 \partial\left(\Delta S^{\prime},|G|\right) \\
& =\partial\left(\Delta S^{\prime} \otimes \mathbb{Z} G, x /|G|^{2}\right)+2 \partial\left(\Delta S^{\prime},|G|\right) .
\end{aligned}
$$

\footnotetext{
$\left({ }^{10}\right)\left(\chi_{1}, \chi_{2}\right)$ denotes the scalar product of the characters $\chi_{1}, \chi_{2}$ of $G$.
} 
The first term has representing homomorphism

$$
\begin{aligned}
\chi & \mapsto \operatorname{det}\left(x /|G|^{2} \mid \operatorname{Hom}_{F G}\left(V_{\chi}, F \otimes\left(\Delta S^{\prime} \otimes \mathbb{Z} G\right)\right)\right) \\
& \doteq \operatorname{det}\left(x /|G|^{2} \mid \operatorname{Hom}_{F G}\left(V_{\chi} \otimes\left(F \otimes \Delta S^{\prime}\right)^{\vee}, F G\right)\right) \\
& =\operatorname{det}\left(x /|G|^{2} \mid V_{\chi} \otimes\left(F \otimes \Delta S^{\prime}\right)^{\vee}\right)
\end{aligned}
$$

by Lemma A.1 in [GRW1]. The equality $\doteq$ holds because of the isomorphism

$$
\operatorname{Hom}_{F}(V, W \otimes Z) \simeq \operatorname{Hom}_{F}\left(V \otimes W^{\vee}, Z\right), \quad t \mapsto[v \otimes \omega \mapsto(\widetilde{\omega} t) v],
$$

where $\omega \in W^{\vee}=\operatorname{Hom}_{F}(W, F)$ induces $\widetilde{\omega}: W \otimes Z \rightarrow Z, w \otimes z \mapsto \omega(w) \cdot z$. This isomorphism respects the $G$-structure and composition by a $G$-endomorphism of $Z$.

Now,

$$
\begin{aligned}
\operatorname{det}\left(x /|G|^{2} \mid V_{\chi} \otimes\left(F \otimes \Delta S^{\prime}\right)^{\vee}\right) & =\prod_{\psi} \operatorname{det}\left(x /|G|^{2} \mid V_{\psi}\right)^{\left(\chi \theta^{\vee}, \psi\right)} \\
& =\prod_{\psi} \psi\left(\frac{x}{|G|^{2}}\right)^{(\chi, \theta \psi)} \\
& =|G|^{-(\chi, \theta)} \prod_{\psi \neq 1}\left(\psi\left(g_{0}\right)-1\right)^{-\left(\chi \psi^{\vee}, \theta\right)} .
\end{aligned}
$$

The second term is represented by

$$
\chi \mapsto \operatorname{det}\left(|G| \mid \operatorname{Hom}_{F G}\left(V_{\chi}, F \otimes \Delta S^{\prime}\right)\right)^{2}=|G|^{2(\chi, \theta)} .
$$

Multiplying the two gives the result.

COROLlary. The Lifted Root Number Conjecture holds for $K / k$ if, and only if, $\left[\mathbb{Z}_{l} \otimes \operatorname{coker} \varphi\right]$ is represented by $\chi \mapsto A_{\varphi}^{(l)}(\check{\chi}) / a_{S^{\prime}}^{(l)}(\chi)$ for all (finite) primes $l$.

The Lifted Root Number Conjecture asserts that $\chi \mapsto A_{\varphi}(\check{\chi})$ represents $\Omega_{\varphi}$, which by Lemmas 3.3 and 3.4 is equivalent to $\chi \mapsto A_{\varphi}(\check{\chi}) / a_{S^{\prime}}(\chi)$ representing $[\operatorname{coker} \varphi]$. This is then restated one prime $l$ at a time by considering the idèlic component above $l$ in the representing homomorphisms [GRW1, Appendix A].

4. Adapting $S$ to the local nature of the Lifted Root Number Conjecture. In the previous section we required $S^{\prime}$ to be sufficiently large in order to have the Tate class $\tau_{S^{\prime}} \in \operatorname{Ext}_{G}^{1}\left(\Delta S^{\prime} \otimes \Delta G, E_{S^{\prime}}\right)$ at our disposal. In this section we restrict $K$ to be absolutely abelian and real, but work with a finite $G$-set $S$ of primes of $K$ containing the set $S_{\infty}$ of infinite primes as well as all ramified primes of the extension $K / k$ and just enough primes to generate the $l$-part of $c l_{K}$ for the given prime $l$. 
Let $n$ denote the conductor of $K$, so $K \subset \mathbb{Q}\left(\zeta_{n}\right)^{+}$, and let $\infty$ be a distinguished infinite prime of $K$. We use the letter $\sigma$ to denote automorphisms of $K / \mathbb{Q}$, so each infinite prime of $K$ is some $\infty^{\sigma}$.

Recall from the introduction the Ramachandra number

$$
\xi_{K}=\prod_{1 \neq d \| n} N_{\mathbb{Q}\left(\zeta_{n}\right) / K}\left(1-\zeta_{n}^{n / d}\right),
$$

with $d \| n$ meaning $d \mid n \&(d, n / d)=1$, and define an $S$-unit $\alpha_{\infty}$ in $K^{\times}$by

$$
\xi_{K}^{g_{0}-1}=\alpha_{\infty}^{\left(g_{0}-1\right)^{h+1}}
$$

with some $h \geq 0$ (as in Lemma 2.2). Moreover, define $\varphi_{\infty}: \Delta S_{\infty} \rightarrow E_{K}$ by $\varphi_{\infty}\left(\infty^{\sigma}-\infty\right)=\alpha_{\infty}^{\sigma-1}$. The comparison of the notation here and in [RW, $\S 10]$ is done by means of the dictionary below.

\begin{tabular}{cc}
\hline RW] & is here \\
\hline$\xi_{K}$ & $\xi_{K}^{2}$ \\
$\varphi_{\infty}$ & $2\left(g_{0}-1\right)^{h} \varphi_{\infty}$ \\
\hline
\end{tabular}

Having thus taken care of all infinite primes of $K$ we get from elements $\alpha_{\mathfrak{p}}\left(\mathfrak{p} \in S_{*}\left({ }^{11}\right), \mathfrak{p} \notin S_{\infty}, \mathfrak{p} \neq \mathfrak{q}_{0}\right)$, as appearing in Proposition 3.2 with $S^{\prime}$ replaced by $S$, a map $\varphi: \Delta S \rightarrow E_{S}$ making the left square of the diagram

$$
\begin{array}{ccccc}
\Delta S_{\infty} & \longmapsto & \Delta S & \rightarrow & \mathbb{Z} S_{f} \\
\downarrow \varphi_{\infty} & & \downarrow \varphi & & \downarrow \widetilde{\varphi} \\
E_{K} & \longmapsto & E_{S} & \rightarrow & E_{S} / E_{K}
\end{array}
$$

commute by sending $\mathfrak{p}-\mathfrak{q}_{0}$ to $\alpha_{\mathfrak{p}}$ and $\infty-\mathfrak{q}_{0}$ to $\alpha_{\infty}$. In the diagram, $S_{f}=S \backslash S_{\infty}, \Delta S \rightarrow \mathbb{Z} S_{f}$ is given by

$$
\mathfrak{p}^{\prime}-\infty \mapsto \begin{cases}\mathfrak{p}^{\prime}, & \mathfrak{p}^{\prime} \text { finite } \\ 0, & \mathfrak{p}^{\prime} \text { infinite },\end{cases}
$$

and the right vertical map $\widetilde{\varphi}$ is the induced one, whence

$$
\widetilde{\varphi}(\mathfrak{p})=\varphi(\mathfrak{p}-\infty) E_{K}=\varphi\left(\mathfrak{p}-\mathfrak{q}_{0}+\mathfrak{q}_{0}-\infty\right) E_{K}=\alpha_{\mathfrak{p}} / \alpha_{\infty} \cdot E_{K}
$$

for $\mathfrak{p} \in S_{*}, \mathfrak{p} \notin S_{\infty}, \mathfrak{p} \neq \mathfrak{q}_{0}$. Similarly, $\widetilde{\varphi}\left(\mathfrak{q}_{0}\right)=\alpha_{\infty}^{-1} E_{K}$.

We define the Dirichlet map $\lambda$ as in [GRW1]: $\lambda_{S}: \mathbb{C} \otimes E_{S} \rightarrow \mathbb{C} \otimes \Delta S$ sends $u \in E_{S}$ to $-\sum_{\mathfrak{p} \in S} \log |u|_{\mathfrak{p}} \mathfrak{p}\left({ }^{12}\right)$. Recall that, for a character $\chi$ of $G$,

$$
A_{\varphi}(\check{\chi})=\frac{\operatorname{det}\left(\lambda_{S} \circ \varphi \mid \operatorname{Hom}_{\mathbb{C} G}\left(V_{\chi}, \mathbb{C} \otimes \Delta S\right)\right)}{c_{S}(\check{\chi})}
$$

is the Tate-Stark number [Ta, p. 27]. We compute it by exploiting our

\footnotetext{
$\left.{ }^{11}\right)$ The $*$ indicates again that $S$ is replaced by a set $S_{*}$ of $G$-representatives.

$\left({ }^{12}\right)$ Observe that this is $-\lambda_{S}$ in [RW].
} 
diagram above which induces

$$
\begin{array}{ccccc}
\mathbb{C} \otimes \Delta S_{\infty} & \longmapsto & \mathbb{C} \otimes \Delta S & \rightarrow & \mathbb{C} S_{f} \\
\downarrow \varphi_{\infty} & & \downarrow \varphi & & \downarrow \widetilde{\varphi} \\
\mathbb{C} \otimes E_{K} & \longmapsto & \mathbb{C} \otimes E_{S} & \rightarrow & \mathbb{C} \otimes E_{S} / E_{K} \\
\downarrow \lambda_{\infty} & & \downarrow \lambda_{S} & & \downarrow \tilde{\lambda} \\
\mathbb{C} \otimes \Delta S_{\infty} & \longmapsto & \mathbb{C} \otimes \Delta S & \rightarrow & \mathbb{C} S_{f}
\end{array}
$$

This implies the factorization

$$
A_{\varphi}(\check{\chi})=\frac{\operatorname{det}\left(\lambda_{\infty} \varphi_{\infty} \mid \operatorname{Hom}_{\mathbb{C} G}\left(V_{\chi}, \mathbb{C} \otimes \Delta S_{\infty}\right)\right)}{c_{S_{\infty}}(\check{\chi})} \cdot \operatorname{det}_{f}(\chi) \cdot \frac{c_{S_{\infty}}(\check{\chi})}{c_{S}(\check{\chi})}
$$

with $\operatorname{det}_{f}(\chi) \operatorname{short}$ for $\operatorname{det}\left(\widetilde{\lambda} \widetilde{\varphi} \mid \operatorname{Hom}_{\mathbb{C} G}\left(V_{\chi}, \mathbb{C} S_{f}\right)\right)$.

The first and third factor have been studied in [RW].

To compute the middle one we use the non-zero elements in $\left\{e_{\chi} \mathfrak{p} \mid\right.$ $\left.\mathfrak{p} \in S_{*}, \mathfrak{p} \notin S_{\infty}\right\}$ as a basis of $\operatorname{Hom}_{\mathbb{C} G}\left(V_{\chi}, \mathbb{C} S_{f}\right)=e_{\chi} \mathbb{C} S_{f}$. Here $e_{\chi}$ is the primitive idempotent corresponding to $\chi$. We have

$$
\tilde{\lambda} \widetilde{\varphi}\left(e_{\chi} \mathfrak{p}\right)=-\sum_{\mathfrak{p}^{\prime} \in S_{f}} \log \left|\frac{\alpha_{\mathfrak{p}}}{\alpha_{\infty}}\right|_{\mathfrak{p}^{\prime}} e_{\chi} \mathfrak{p}^{\prime}
$$

and will evaluate such determinants in Section 5 by applying the

Lemma 4.1. If $S_{f *}$ is a set of $G$-representatives in $S_{f}$, and if $(\alpha)=$ $\prod_{\mathfrak{p} \in S_{f *}} \mathfrak{p}^{x_{\mathfrak{p}}}$ with $x_{\mathfrak{p}} \in \mathbb{Z} G$, then

$$
-\sum_{\mathfrak{p} \in S_{f}} \log |\alpha|_{\mathfrak{p}} e_{\chi} \mathfrak{p}=\sum_{\mathfrak{p} \in S_{f *}} \chi\left(x_{\mathfrak{p}}\right) \log (N \mathfrak{p}) e_{\chi} \mathfrak{p} .
$$

For the proof pick a $\mathfrak{p} \in S_{f *}$ and consider the orbit sum

$$
-\sum_{\mathfrak{p}^{\prime} \in G \cdot \mathfrak{p}} \log |\alpha|_{\mathfrak{p}^{\prime}} e_{\chi} \mathfrak{p}^{\prime}=-\frac{1}{\left|G_{\mathfrak{p}}\right|} \sum_{g \in G} \log \left|\alpha^{g^{-1}}\right|_{\mathfrak{p}} \chi(g) e_{\chi} \mathfrak{p} .
$$

With $\kappa: \mathbb{C} G \rightarrow \mathbb{C}$ denoting the $\mathbb{C}$-linear map taking $g$ to 1 or 0 according as $g \in G_{\mathfrak{p}}$ or $g \notin G_{\mathfrak{p}}$ we deduce from $v_{\mathfrak{p}}\left(\alpha^{g^{-1}}\right)=\kappa\left(g^{-1} x_{\mathfrak{p}}\right)$ that the above orbit sum equals

$$
\begin{aligned}
-\frac{1}{\left|G_{\mathfrak{p}}\right|} \sum_{g \in G} & \left(-\kappa\left(g^{-1} x_{\mathfrak{p}}\right) \log (N \mathfrak{p})\right) \chi(g) e_{\chi} \mathfrak{p} \\
& =\frac{\log (N \mathfrak{p})}{\left|G_{\mathfrak{p}}\right|} \kappa\left(\sum_{g \in G} \chi(g) g^{-1} x_{\mathfrak{p}}\right) e_{\chi} \mathfrak{p}=\frac{\log (N \mathfrak{p})}{\left|G_{\mathfrak{p}}\right|} \kappa\left(|G| e_{\chi} x_{\mathfrak{p}}\right) e_{\chi} \mathfrak{p}
\end{aligned}
$$




$$
\begin{aligned}
& =\frac{\log (N \mathfrak{p})}{\left|G_{\mathfrak{p}}\right|} \kappa\left(\chi\left(x_{\mathfrak{p}}\right)|G| e_{\chi}\right) e_{\chi} \mathfrak{p} \\
& =\frac{\log (N \mathfrak{p})}{\left|G_{\mathfrak{p}}\right|} \chi\left(x_{\mathfrak{p}}\right)\left(\sum_{g \in G_{\mathfrak{p}}} \chi(g)\right) e_{\chi} \mathfrak{p} \\
& =\frac{\log (N \mathfrak{p})}{\left|G_{\mathfrak{p}}\right|} \chi\left(x_{\mathfrak{p}}\right)\left|G_{\mathfrak{p}}\right| e_{\chi} \mathfrak{p},
\end{aligned}
$$

since $e_{\chi} \mathfrak{p}=0$ whenever $\operatorname{res}_{G_{\mathfrak{p}}}^{G} \chi$ is non-trivial.

LEMMA 4.2. Even though $S$ does not satisfy the hypothesis of Proposition 3.2, the Corollary at the end of Section 3 remains true for $S$ with respect to the given prime $l$.

To see this, let $S^{\prime}$ be a finite $G$-set containing $S$ and $G$-orbits of split primes $\mathfrak{r}$ (over $k$ ) so that the $S^{\prime}$-class group of $K$ vanishes. The existence of an $S^{\prime}$ follows from the Chebotarev density theorem.

Assume now that we have a map $\varphi: \Delta S \longmapsto E_{S}$ satisfying $\varphi\left(\mathfrak{p}-\mathfrak{q}_{0}\right)=\alpha_{\mathfrak{p}}$ for $\mathfrak{p} \in S_{*}, \mathfrak{p} \neq \mathfrak{q}_{0}$, where the $\alpha_{\mathfrak{p}}$ are as above $\left(\mathfrak{p}=\infty\right.$ included). With $h^{\prime}$ denoting the $l$-prime part of $\left|c l_{K}\right|$, we pick generators $\alpha_{\mathfrak{r}} \in K$ of the principal ideals $\mathfrak{r}^{h^{\prime}}$ and extend $\varphi$ to $\varphi^{\prime}: \Delta S^{\prime} \rightarrow E_{S^{\prime}}$ by mapping $\mathfrak{r}-\mathfrak{q}_{0}$ to $\alpha_{\mathfrak{r}}$. Then $\varphi^{\prime}$ is a map as described in Proposition 3.2, because the $\mathfrak{r}$ split over $k$ and since the $\alpha_{\mathfrak{r}}$ are $S^{\prime}$-units.

We first compare coker $\varphi$ and coker $\varphi^{\prime}$. To do so build

$$
\begin{array}{cccccc}
\Delta S & \longmapsto & \Delta S^{\prime} & \rightarrow & \mathbb{Z}\left[S^{\prime} \backslash S\right] \\
\downarrow \varphi & & \downarrow \varphi^{\prime} & & \\
E_{S} & \longmapsto & E_{S^{\prime}} & \rightarrow & E_{S^{\prime}} / E_{S} \\
& & & & \downarrow \\
& & & & \mathbb{Z}\left[S^{\prime} \backslash S\right]
\end{array}
$$

analogous to the earlier diagram (with $S_{\infty}, S$ instead of $S, S^{\prime}$ ) and with $E_{S^{\prime}} / E_{S} \rightarrow \mathbb{Z}\left[S^{\prime} \backslash S\right]$ taking $u \in E_{S^{\prime}}$ to $\sum_{\mathfrak{r}} v_{\mathfrak{r}}(u) \mathfrak{r}$. The composite right vertical map is then multiplication by $h^{\prime}: \mathfrak{r} \mapsto \alpha_{\mathfrak{r}} \mapsto h^{\prime} \mathfrak{r}$. Hence $\mathbb{Z}_{l} \otimes$ $\operatorname{coker} \varphi=\mathbb{Z}_{l} \otimes \operatorname{coker} \varphi^{\prime}$.

We next turn to the numerators of the $A$-numbers. We tensor the above diagram with $\mathbb{C}$ and get a diagram similar to (4.1) with maps $\varphi, \varphi^{\prime}, \varphi^{\prime \prime}$ and $\lambda, \lambda^{\prime}, \lambda^{\prime \prime}$, say. Thus

$$
\operatorname{det}(\lambda \varphi)=\operatorname{det}\left(\lambda^{\prime} \varphi^{\prime}\right) / \operatorname{det}\left(\lambda^{\prime \prime} \varphi^{\prime \prime}\right)=\operatorname{det}\left(\lambda^{\prime} \varphi^{\prime}\right) / \prod_{\mathfrak{r}}\left(h^{\prime} \log N \mathfrak{r}\right)
$$

by $\lambda^{\prime \prime} \varphi^{\prime \prime}(\mathfrak{r})=h^{\prime}(\log N \mathfrak{r}) \mathfrak{r}$.

Now,

$$
\frac{A_{\varphi^{\prime}}(\check{\chi})}{A_{\varphi}(\check{\chi})}=\frac{c_{S}(\check{\chi})}{c_{S^{\prime}}(\check{\chi})} \cdot \prod_{\mathfrak{r}} h^{\prime} \log (N \mathfrak{r})=\prod_{\mathfrak{r}} h^{\prime}
$$

by [RW, Lemma 7 with $\left.G_{\mathfrak{r}}=I_{\mathfrak{r}}=1, f_{\mathfrak{r}}=1\right]$. 
Finally we look at $a_{S^{\prime}}(\chi) / a_{S}(\chi)$. As the character of $\mathbb{Z}\left[S^{\prime} \backslash S\right]$ is a multiple of the regular character $\varrho$, we get from Lemma 3.4, for irreducible $\chi$,

$$
\frac{a_{S^{\prime}}(\chi)}{a_{S}(\chi)}=\prod_{\mathfrak{r}}\left(|G|^{(\chi, \varrho)} \prod_{\psi \neq 1}\left(\psi\left(g_{0}\right)-1\right)^{-\left(\chi \psi^{-1}, \varrho\right)}\right)=\prod_{\mathfrak{r}}(-1)^{|G|-1},
$$

because the multiplicity in $\varrho$ of every irreducible character is 1 .

Putting things together, we see that $A_{\varphi^{\prime}}(\chi) / a_{S^{\prime}}(\chi)$ and $A_{\varphi}(\check{\chi}) / a_{S}(\chi)$ differ by a constant $b \in \mathbb{Z}_{l}^{\times}$which is independent of the irreducible character $\chi$. Since $\chi \mapsto b^{\chi(1)} \in \operatorname{Det}\left(\mathbb{Z}_{l} G\right)$ represents the trivial element in $K_{0} T\left(\mathbb{Z}_{l} G\right)$, the lemma is proved.

5. Calculation of $A_{\varphi}$. We go back to our initial situation in which $K$ is as in the introduction.

With a choice of $\mathfrak{q}_{0}, \ldots, \mathfrak{q}_{r-1}$ as in Lemma 2.3, suppose that $\mathfrak{a}_{i}, 1 \leq$ $i \leq r-1$, are ideals supported in $\left\{\mathfrak{p}_{1}, \ldots, \mathfrak{p}_{r}\right\}$ such that $\mathfrak{a}_{i} \cdot \mathfrak{q}_{i}^{\left(g_{0}-1\right)^{h_{i}-1}}$ are principal. Let $\alpha_{\mathfrak{q}_{i}}$ be the corresponding generators.

LEMMA 5.1. The $r \times r$ matrix

$$
\left(\begin{array}{ccc}
-v_{\mathfrak{p}_{1}}\left(\alpha_{\infty}\right) & \ldots & -v_{\mathfrak{p}_{r}}\left(\alpha_{\infty}\right) \\
-v_{\mathfrak{p}_{1}}\left(\alpha_{\mathfrak{q}_{1}}\right) & \ldots & -v_{\mathfrak{p}_{r}}\left(\alpha_{\mathfrak{q}_{1}}\right) \\
\vdots & \ldots & \vdots \\
-v_{\mathfrak{p}_{1}}\left(\alpha_{\mathfrak{q}_{r-1}}\right) & \ldots & -v_{\mathfrak{p}_{r}}\left(\alpha_{\mathfrak{q}_{r-1}}\right)
\end{array}\right)
$$

is non-singular modulo $l$. Moreover, its determinant $c^{\prime}$ satisfies $-v_{\mathfrak{p}_{j}}\left(\alpha_{\infty}\right) \equiv$ $c^{\prime} B_{j} \bmod l$ for $1 \leq j \leq r$. In other words, $c^{\prime} \equiv \widetilde{c} \bmod l$.

In fact, $\left(\alpha_{\mathfrak{q}_{i}}\right)=\mathfrak{q}_{i}^{\left(g_{0}-1\right)^{h_{i}-1}} \prod_{s=1}^{r} \mathfrak{p}_{s}^{v_{\mathfrak{p}_{s}}\left(\alpha_{\mathfrak{q}_{i}}\right)}$ and (1.2) imply

$$
\sum_{s=1}^{r}\left(-v_{\mathfrak{p}_{s}}\left(\alpha_{\mathfrak{q}_{i}}\right)\right) b_{j s} \equiv \delta_{i j} \bmod l, \quad 1 \leq i, j \leq r-1 .
$$

Since the $B_{j}$ and $v_{\mathfrak{p}_{j}}\left(\alpha_{\infty}\right)$ are proportional modulo $l$, we also have

$$
\sum_{s=1}^{r}\left(-v_{\mathfrak{p}_{j}}\left(\alpha_{\infty}\right)\right) b_{j s} \equiv 0 \bmod l \quad \text { for } 1 \leq j \leq r-1 .
$$

With $\left[\begin{array}{c}z \\ b_{i j}\end{array}\right]$ denoting the matrix

$$
\left(\begin{array}{lll}
z_{1} & \ldots & z_{r} \\
& b_{i j} &
\end{array}\right)_{\substack{1 \leq j \leq r \leq r \\
1 \leq i \leq r-1}},
$$

where $\left(z_{1}, \ldots, z_{r}\right)$ is any row vector, we see that the matrix in the statement 
of the lemma times the transpose of $\left[\begin{array}{c}z \\ b_{i j}\end{array}\right]$ is

$$
\left(\begin{array}{cccc}
\sum_{j=1}^{r}\left(-v_{\mathfrak{p}_{j}}\left(\alpha_{\infty}\right)\right) z_{j} & 0 & \ldots & 0 \\
* & 1 & & \\
\vdots & & & \\
* & & & 1
\end{array}\right) .
$$

Taking determinants yields $c^{\prime} \cdot\left(\sum_{j=1}^{r} z_{j} B_{j}\right) \equiv \sum_{j=1}^{r}\left(-v_{\mathfrak{p}_{j}}\left(\alpha_{\infty}\right)\right) z_{j} \bmod l$ by the definition of the $B_{j}$. The second assertion of Lemma 5.1 follows by varying $z$ and then the first assertion from Lemma 2.2 .

In the vacuous case $r=1$ we see that we had to set $B_{1}=1$.

Corollary. For each $1 \leq j \leq r, p_{j}$ is in the $\mathbb{Z}_{l} G$-span of the $q_{i}$ and the $\alpha_{\mathfrak{q}_{i}}, \alpha_{\infty}$.

For $\alpha_{\mathfrak{q}_{i}}^{\widehat{G}}=q_{i}^{\delta_{i}} p_{1}^{v_{\mathfrak{p}_{1}}\left(\alpha_{\mathfrak{q}_{i}}\right)} \ldots p_{r}^{v_{\mathfrak{p}_{r}}\left(\alpha_{\mathfrak{q}_{i}}\right)}$ in $\mathbb{Z}_{l} \otimes E_{S}$, with $\delta_{i}=0$ or 1 according as $h_{i}>1$ or $h_{i}=1$, and similarly for $\alpha_{\infty}$.

We use the $\alpha_{\mathfrak{q}_{i}}$ and $\alpha_{\infty}$ together with an integer matrix $\left(x_{i j}\right)$ congruent modulo $l$ to the inverse of the matrix $\left(c_{i j}\right)$ of Lemma 2.3 in order to define a $G$-map $\varphi: \Delta S \rightarrow E_{S}$, where $S$ is the $G$-set generated by $\left\{\infty, \mathfrak{p}_{1}, \ldots, \mathfrak{p}_{r}, \mathfrak{q}_{0}, \ldots, \mathfrak{q}_{r-1}\right\}$. Here, $\infty$ is the infinite prime defined by $K \subset \mathbb{Q}\left(\zeta_{n}\right) \hookrightarrow \mathbb{C}, \zeta_{n} \mapsto e^{2 \pi i / n}$.

$\Delta S$ is spanned over $\mathbb{Z} G$ by

$$
\mathfrak{p}_{j}-\mathfrak{q}_{0}, 1 \leq j \leq r, \quad \mathfrak{q}_{i}-\mathfrak{q}_{0}, 1 \leq i \leq r-1, \quad \infty-\mathfrak{q}_{0}
$$

$G$ acts trivially on the $\mathfrak{p}_{j}-\mathfrak{q}_{0}$; the other generators are free over $\mathbb{Z} G$. The map $\varphi$ is defined by

$$
\mathfrak{p}_{j}-\mathfrak{q}_{0} \mapsto \prod_{i=0}^{r-1} q_{i}^{x_{j i}}=: \alpha_{\mathfrak{p}_{j}}, \quad \mathfrak{q}_{i}-\mathfrak{q}_{0} \mapsto \alpha_{\mathfrak{q}_{i}}, \quad \infty-\mathfrak{q}_{0} \mapsto \alpha_{\infty}
$$

Lemma 5.2. $\varphi$ satisfies the conditions of Proposition 3.2 and induces an $\mathbb{Z}_{l} G$-isomorphism $\mathbb{Z}_{l} \otimes \Delta S \rightarrow \mathbb{Z}_{l} \otimes E_{S}$.

Proof. Because $\mathfrak{q}_{1}, \ldots, \mathfrak{q}_{r-1}, \infty$ are split over $\mathbb{Q}$ there is no condition on the $\alpha_{\mathfrak{q}_{i}}$ and on $\alpha_{\infty}$ in Proposition 3.2. Concerning the $\alpha_{\mathfrak{p}_{j}}$ we have

$$
\left(\alpha_{\mathfrak{p}_{j}}, K_{\mathfrak{p}_{j}^{\prime}} / \mathbb{Q}_{p_{j}^{\prime}}\right)=\prod_{i=0}^{r-1}\left(\mathfrak{q}_{i}, K_{\mathfrak{p}_{j}^{\prime}} / \mathbb{Q}_{p_{j}^{\prime}}\right)^{x_{j i}}=g_{0}^{\sum_{i} x_{j i} c_{i j^{\prime}}}=g_{0}^{\delta_{j j^{\prime}}} .
$$

Moreover, $\left(\alpha_{\mathfrak{p}_{j}}, K_{\mathfrak{p}} / \mathbb{Q}_{p}\right)=1$ for all $p \neq q_{0}, p_{1}, \ldots, p_{r}$, since either $p$ splits or $\alpha_{\mathfrak{p}_{j}}$ is a unit in the unramified extension $K_{\mathfrak{p}} / \mathbb{Q}_{p}$.

To see that $\varphi$ is an $l$-adic isomorphism we show first that $q_{0}, q_{1}, \ldots, q_{r-1}$, $\alpha_{\infty}, \alpha_{\mathfrak{q}_{1}}, \ldots, \alpha_{\mathfrak{q}_{r-1}}$ generate $\mathbb{Z}_{l} \otimes E_{S}$ as $\mathbb{Z}_{l} G$-module. Pick $u \in E_{S}$. Then, by definition of $S$,

$$
(u)=\mathfrak{p}_{1}^{b_{1}} \ldots \mathfrak{p}_{r}^{b_{r}} \mathfrak{q}_{0}^{c_{0}} \ldots \mathfrak{q}_{r-1}^{c_{r-1}}
$$


with $b_{j} \in \mathbb{Z}$ and $c_{i} \in \mathbb{Z} G$. Because $q_{0}$ is inert and $q_{0} \in E_{S}$, we may assume $c_{0}=0$. Reading then the above equation in $c l_{K}$ shows $\mathfrak{C}_{1}^{c_{1}} \ldots \mathfrak{C}_{r-1}^{c_{r-1}} \in c l_{K}^{G}$ and so $c_{i}=c_{i}^{\prime}\left(g_{0}-1\right)^{h_{i}-1}+c_{i}^{\prime \prime} \widehat{G}$ with $c_{i}^{\prime} \in \mathbb{Z} G$ and $c_{i}^{\prime \prime} \in \mathbb{Z}$.

It follows from the definition of the $\alpha_{\mathfrak{q}_{i}}$ that

$$
\left(u \prod_{i=1}^{r-1} \alpha_{\mathfrak{q}_{i}}^{-c_{i}^{\prime}}\right)=\mathfrak{p}_{1}^{b_{1}^{\prime}} \ldots \mathfrak{p}_{r}^{b_{r}^{\prime}} \prod_{i=1}^{r-1} q_{i}^{c_{i}^{\prime \prime}} .
$$

So $\mathfrak{p}_{1}^{b_{1}^{\prime}} \ldots \mathfrak{p}_{r}^{b_{r}^{\prime}}$ is principal, whence $\left(\mathfrak{p}_{1}^{b_{1}^{\prime}} \ldots \mathfrak{p}_{r}^{b_{r}^{\prime}}\right)^{N}=\left(\alpha_{\infty}^{d} a\right)$ with $d \in \mathbb{Z}, a$ a product of the $p_{j}$ and with a suitable $N \not \equiv 0 \bmod l$. Therefore $u^{N}$ and $\alpha_{\infty}^{d} a \prod_{i=1}^{r-1}\left(\alpha_{q_{i}}^{N c_{i}^{\prime}} q_{i}^{N c_{i}^{\prime \prime}}\right)$ differ by a unit. Read in $\mathbb{Z}_{l} \otimes E_{S}$, this unit becomes a $\mathbb{Z}_{l} G$-power of $\alpha_{\infty}^{g_{0}-1}$ by Lemma 2.2. Because of the above corollary and since $l \nmid N$, our generation claim is proved.

The non-degeneracy of the matrix $\left(x_{i j}\right)$ modulo $l$ then implies that $\varphi$ is surjective modulo $l$, so $l$-adically as well. This finishes the proof by Dirichlet's unit theorem.

We now turn to the computation of $A_{\varphi}(\check{\chi})$. Because of (4.2) this amounts to computing the three quantities

$$
\frac{\operatorname{det}\left(\lambda_{\infty} \varphi_{\infty} \mid \operatorname{Hom}_{G}\left(V_{\chi}, \Delta S_{\infty}\right)\right)}{c_{S_{\infty}}(\check{\chi})}, \quad \operatorname{det}_{f}(\chi) \text { and } \frac{c_{S_{\infty}}(\check{\chi})}{c_{S}(\check{\chi})},
$$

where $\varphi$ is decomposed according to the diagram

$$
\begin{array}{ccccc}
\Delta S_{\infty} & \longmapsto & \Delta S & \rightarrow & \mathbb{Z} S_{f} \\
\downarrow \varphi_{\infty} & & \downarrow \varphi & & \downarrow \widetilde{\varphi} \\
E_{K} & \longmapsto & E_{S} & \rightarrow & E_{S} / E_{K}
\end{array}
$$

with $\varphi_{\infty}\left(\infty^{g}-\infty\right)=\alpha_{\infty}^{g-1}(g \in G)$, and where $\operatorname{det}_{f}(\chi)$ is the determinant of the map $e_{\chi} \mathbb{C} S_{f} \rightarrow e_{\chi} \mathbb{C} S_{f}$ taking:

- $e_{\chi} \mathfrak{p}_{j}(1 \leq j \leq r)$ to

$$
\begin{aligned}
-\sum_{\mathfrak{p} \in S_{f}}\left(\log \left|\frac{\alpha_{\mathfrak{p}_{j}}}{\alpha_{\infty}}\right|_{\mathfrak{p}}\right) e_{\chi} \mathfrak{p}= & -\sum_{j=1}^{r} v_{\mathfrak{p}_{j}}\left(\alpha_{\infty}\right)\left(\log p_{j}\right) e_{\chi} \mathfrak{p}_{j} \\
& +\sum_{i=0}^{r-1} \chi(\widehat{G}) x_{j i}\left(\log q_{i}\right) e_{\chi} \mathfrak{q}_{i}
\end{aligned}
$$

by Lemma 4.1, since $\alpha_{\mathfrak{p}_{j}}=\prod_{i=0}^{r-1} q_{i}^{x_{j i}}, q_{i}=\mathfrak{q}_{i}^{\widehat{G}}(1 \leq i \leq r-1), N\left(\mathfrak{q}_{0}\right)=q_{0}^{l}$ and $e_{\chi} \mathfrak{q}_{0}=0$ if $\chi \neq 1$, and since $\alpha_{\infty}$ is supported in $\left\{\mathfrak{p}_{1}, \ldots, \mathfrak{p}_{r}\right\}$;

- $e_{\chi} \mathfrak{q}_{0}$ to

$$
-\sum_{\mathfrak{p} \in S_{f}}\left(\log \left|\alpha_{\infty}^{-1}\right|_{\mathfrak{p}}\right) e_{\chi} \mathfrak{p}=-\sum_{j=1}^{r} v_{\mathfrak{p}_{j}}\left(\alpha_{\infty}\right)\left(\log p_{j}\right) e_{\chi} \mathfrak{p}_{j}
$$


- $e_{\chi} \mathfrak{q}_{i}(1 \leq i \leq r-1)$ to

$$
\sum_{j=1}^{r} v_{\mathfrak{p}_{j}}\left(\alpha_{\mathfrak{q}_{i}} \alpha_{\infty}^{-1}\right)\left(\log p_{j}\right) e_{\chi} \mathfrak{p}_{j}+\left(\chi\left(g_{0}\right)-1\right)^{h_{i}-1}\left(\log q_{i}\right) e_{\chi} \mathfrak{q}_{i}
$$

since $\left(\alpha_{\mathfrak{q}_{i}}\right)=\mathfrak{q}_{i}^{\left(g_{0}-1\right)^{h_{i}-1}} \mathfrak{a}_{i}$.

Regarding the first quantity we have

$$
\frac{\operatorname{det}_{\chi}\left(\lambda_{\infty} \varphi_{\infty}\right)}{c_{S_{\infty}}(\check{\chi})}= \begin{cases}-2 & \text { if } \chi=1 \\ 2 /\left(\chi\left(g_{0}\right)-1\right)^{h} & \text { if } \chi \neq 1\end{cases}
$$

This follows from $e_{1} \Delta S_{\infty}=0, c_{S_{\infty}}(1)=-1 / 2$ and from Proposition 12 of [RW] adjusted appropriately (see $\S 4$ ); in particular, the $\lambda_{\infty}$ there is here $-\lambda_{\infty}$.

The quantity $c_{S_{\infty}}(\check{\chi}) / c_{S}(\check{\chi})$ is given by [RW, Lemma 7] on observing

$$
\begin{aligned}
& \check{V}^{G_{\mathfrak{p}_{j}}}=0=\check{V}^{I_{\mathfrak{p}_{j}}}(1 \leq j \leq r), \quad \check{V}^{G_{\mathfrak{q}_{i}}}=\check{V}=\check{V}^{I_{\mathfrak{q}_{i}}}(1 \leq i \leq r-1), \\
& \check{V}^{G_{\mathrm{q}_{0}}}=0, \quad \check{V}^{I_{\mathrm{q}_{0}}}=\check{V},
\end{aligned}
$$

where $\check{V}$ is a $\mathbb{C} G$-module affording the character $\check{\chi} \neq 1$, and where $I_{\mathfrak{p}}$, for a prime $\mathfrak{p}$, is the inertia group of $\mathfrak{p}$. Thus

$$
\begin{aligned}
& \frac{c_{S_{\infty}}(\check{\chi})}{c_{S}(\check{\chi})} \\
= & \begin{cases}\prod_{j=1}^{r}\left(\log p_{j}\right)^{-1} \prod_{i=0}^{r-1}\left(\log q_{i}\right)^{-1} & \text { for } \chi=1, \\
\left(\left(\prod_{i=1}^{r-1} \log q_{i}\right)\left(1-\check{\chi}\left(g_{0}\right)\right)\right)^{-1}=\frac{\chi\left(g_{0}\right)}{\chi\left(g_{0}\right)-1} \prod_{i=1}^{r-1}\left(\log q_{i}\right)^{-1} & \text { for } \chi \neq 1 .\end{cases}
\end{aligned}
$$

We are left with computing $\operatorname{det}_{f}(\chi)$. Assume first $\chi \neq 1$. Then $e_{\chi} \mathfrak{p}_{j}=$ $0=e_{\chi} \mathfrak{q}_{0}, 1 \leq j \leq r$, and our map $e_{\chi} \mathbb{C} S_{f} \rightarrow e_{\chi} \mathbb{C} S_{f}$ is diagonal with diagonal entries $\left(\chi\left(g_{0}\right)-1\right)^{h_{i}-1}\left(\log q_{i}\right)$, so

$$
\operatorname{det}_{f}(\chi)=\left(\chi\left(g_{0}\right)-1\right)^{\sum_{i=1}^{r-1} h_{i}-(r-1)} \prod_{i=1}^{r-1}\left(\log q_{i}\right) .
$$

Assume next that $\chi=1$. We label the row and columns of the matrix of $e_{1} \mathbb{C} S_{f} \rightarrow e_{1} \mathbb{C} S_{f}$ by $e_{1} \mathfrak{p}_{j}(1 \leq j \leq r), e_{1} \mathfrak{q}_{i}(0 \leq i \leq r-1)$ starting at the top and on the left, respectively. Then we view it as having the form $\left(\begin{array}{ll}A_{11} & A_{12} \\ A_{21} & A_{22}\end{array}\right)$ with $r \times r$ matrices $A_{\nu \mu}$. Subtracting row $e_{1} \mathfrak{q}_{0}$ from all the other rows changes $A_{11}$ into the zero matrix but does not affect $A_{12}$ as follows 
from the description of the map $e_{\chi} \mathbb{C} S_{f} \rightarrow e_{\chi} \mathbb{C} S_{f}$. However, $A_{21}$ becomes

$$
A_{21}^{\prime}=\left(\begin{array}{ccc}
-v_{\mathfrak{p}_{1}}\left(\alpha_{\infty}\right) \log p_{1} & \ldots & -v_{\mathfrak{p}_{r}}\left(\alpha_{\infty}\right) \log p_{r} \\
v_{\mathfrak{p}_{1}}\left(\alpha_{\mathfrak{q}_{1}}\right) \log p_{1} & \ldots & v_{\mathfrak{p}_{r}}\left(\alpha_{\mathfrak{q}_{1}}\right) \log p_{r} \\
\vdots & & \vdots \\
v_{\mathfrak{p}_{1}}\left(\alpha_{\mathfrak{q}_{r-1}}\right) \log p_{1} & \ldots & v_{\mathfrak{p}_{r}}\left(\alpha_{\mathfrak{q}_{r-1}}\right) \log p_{r}
\end{array}\right)
$$

Hence

$$
\begin{aligned}
\operatorname{det}_{f}(1) & =(-1)^{r} \operatorname{det} A_{12} \operatorname{det} A_{21}^{\prime} \\
& =(-1)^{r} \operatorname{det} A_{12} \cdot\left(\prod_{j=1}^{r} \log p_{j}\right) \cdot(-1) \cdot \operatorname{det}\left(v_{\mathfrak{p}_{j}}\left(\alpha_{\mathfrak{q}_{i}}\right)\right),
\end{aligned}
$$

where in accordance with our usual numbering we better read $\alpha_{\infty}$ as $\alpha_{\mathfrak{q}_{0}}$.

As for $\operatorname{det} A_{12}$, we remember that

$$
A_{12}=\left(\begin{array}{ccc}
l x_{10} \log q_{0} & \ldots & l x_{1, r-1} \log q_{r-1} \\
\vdots & & \vdots \\
l x_{r 0} \log q_{0} & \ldots & l x_{r, r-1} \log q_{r-1}
\end{array}\right),
$$

so

$$
\operatorname{det} A_{12}=l^{r}\left(\prod_{i=0}^{r-1} \log q_{i}\right) \operatorname{det}\left(x_{j i}\right) .
$$

Taking everything into account we arrive at

$$
= \begin{cases}(-1) l^{r}\left(\prod_{j=1}^{r} \log p_{j}\right)\left(\prod_{i=0}^{r-1} \log q_{i}\right) \operatorname{det}\left(-v_{\mathfrak{p}_{j}}\left(\alpha_{\mathfrak{q}_{i}}\right)\right) \operatorname{det}\left(x_{j i}\right) & \text { if } \chi=1, \\ \left(\prod_{i=1}^{r-1} \log q_{i}\right)\left(\chi\left(g_{0}\right)-1\right)^{\sum_{i=1}^{r-1} h_{i}-(r-1)} & \text { if } \chi \neq 1 .\end{cases}
$$

(5.1)-(5.3) together yield

$$
\begin{aligned}
& A_{\varphi}(\check{\chi}) \\
& = \begin{cases}2 l^{r} \operatorname{det}\left(-v_{\mathfrak{p}_{j}}\left(\alpha_{\mathfrak{q}_{i}}\right)\right) \operatorname{det}\left(x_{j i}\right) & \text { if } \chi=1, \\
\frac{2}{\left(\chi\left(g_{0}\right)-1\right)^{h}} \cdot \frac{\chi\left(g_{0}\right)}{\chi\left(g_{0}\right)-1}\left(\chi\left(g_{0}\right)-1\right)^{\sum_{i=1}^{r-1} h_{i}} /\left(\chi\left(g_{0}\right)-1\right)^{r-1} & \text { if } \chi \neq 1 .\end{cases}
\end{aligned}
$$

Using the decomposition $\mathbb{C} \otimes \Delta S=\mathbb{C}^{r} \oplus(\mathbb{C} G)^{r}$ we quickly evaluate the 
representing homomorphism $a(\chi)$ from Lemma 3.4:

$$
a(\chi)=\left\{\begin{array}{cc}
l^{2 r} \prod_{\psi \neq 1}\left(\psi\left(g_{0}\right)-1\right)^{-\left(\psi^{-1}, \Delta S\right)}=\frac{l^{2 r}}{\prod_{\psi \neq 1}\left(\psi\left(g_{0}\right)-1\right)^{r}}=l^{r}, \quad \chi=1, \\
l^{r}\left(\chi\left(g_{0}\right)-1\right)^{-2 r} \prod_{\psi \neq 1, \chi}\left(\psi\left(g_{0}\right)-1\right)^{r}=\frac{l^{r}\left(\chi\left(g_{0}\right)-1\right)^{-r}}{\prod_{\psi \neq 1}\left(\psi\left(g_{0}\right)-1\right)^{r}} \\
=\left(\chi\left(g_{0}\right)-1\right)^{-r}, \quad \chi \neq 1 .
\end{array}\right.
$$

Therefore,

$$
\frac{A_{\varphi}(\check{\chi})}{a(\chi)}= \begin{cases}2 \operatorname{det}\left(-v_{\mathfrak{p}_{j}}\left(\alpha_{\mathfrak{q}_{i}}\right)\right) \operatorname{det}\left(x_{j i}\right) & \text { if } \chi=1 \\ \frac{2}{\left(\chi\left(g_{0}\right)-1\right)^{h}}\left(\chi\left(g_{0}\right)-1\right)^{\sum_{i=1}^{r-1} h_{i}} \cdot \chi\left(g_{0}\right) & \text { if } \chi \neq 1\end{cases}
$$

As has been pointed out at the end of Section $3, \chi \mapsto A_{\varphi}^{(l)}(\check{\chi}) / a^{(l)}(\chi)$ is to represent $\mathbb{Z}_{l} \otimes \operatorname{coker} \varphi=0$ by the Lifted Root Number Conjecture. The main result in $[\mathrm{RW}]$ confirms this modulo $D T\left(\mathbb{Z}_{l} G\right)$, i.e., all $A_{\varphi}(\check{\chi}) / a(\chi)$ generate the same ideal in $\mathbb{Z}_{l}\left[\zeta_{l}\right]$. In particular,

$$
\sum_{i=1}^{r-1} h_{i}=h
$$

since $\operatorname{det}\left(-v_{\mathfrak{p}_{j}}\left(\alpha_{\mathfrak{q}_{i}}\right)\right) \cdot \operatorname{det}\left(x_{j i}\right) \not \equiv 0 \bmod l$ by Lemmas 2.3 and 5.1. Consequently, the Lifted Root Number Conjecture amounts to

$$
A_{\varphi}(\check{\chi}) / a(\chi) \equiv A_{\varphi}(1) / a(1) \bmod \chi\left(g_{0}\right)-1
$$

(for all $\chi \neq 1$ ), by [GRW1, Proposition 8(iii)], that is

$$
\operatorname{det}\left(-v_{\mathfrak{p}_{j}}\left(\alpha_{\mathfrak{q}_{i}}\right)\right) \equiv \operatorname{det}\left(c_{i j}\right) \bmod l
$$

by Lemma 2.3. This is indeed conjecture (C), by Lemma 5.1 .

We quickly dispose of the case $r=1$, whence $h=0$ by ( $($ ) $)$. First of all, $c_{01}=-1$ by the reciprocity law. In fact, $\left(q_{0}, K_{\mathfrak{p}_{1}} / \mathbb{Q}_{p_{1}}\right)=g_{0}^{c_{01}},\left(q_{0}, K_{\mathfrak{q}_{0}} / \mathbb{Q}_{q_{0}}\right)$ $=g_{0}$, and $\left(q_{0}, K_{\mathfrak{p}} / \mathbb{Q}_{p}\right)=1$ for all other primes $p$, since these are unramified. Moreover, $K$ is the subfield of $\mathbb{Q}\left(\zeta_{p_{1}}\right)$ of degree $l$ over $\mathbb{Q}$ and so $\xi_{K}$ is a prime element for $\mathfrak{p}_{1}$. By Lemma 2.2 we can take $\alpha_{\infty}=\xi_{K}$. Hence, (C) and (LC) both say $-1 \equiv-1 \bmod l$.

From now on, we assume that $r \geq 2$.

We close this section with an observation concerning the $\mathfrak{p}_{j}$-value of an element $\alpha \in K^{\times}$and the congruence class of $\alpha^{g_{0}-1}$ modulo $\mathfrak{p}_{j}$. To do so, we first define

$$
m_{j}=\frac{p_{j}-1}{l}, \quad 1 \leq j \leq r,
$$

and recall that $\left(q_{0}, K_{\mathfrak{p}_{j}} / \mathbb{Q}_{p_{j}}\right)=g_{0}^{c_{0 j}}$. 
Lemma 5.3. The $\mathfrak{p}_{j}$-value $a_{j}$ of an element $\alpha \in K^{\times}$is determined modulo $l$ by the congruence

$$
\alpha^{c_{0 j}\left(g_{0}-1\right)} \equiv q_{0}^{-m_{j} a_{j}} \bmod \mathfrak{p}_{j} .
$$

For the proof we view all occurring quantities as elements of the completion $K_{\mathfrak{p}_{j}}$, which is a totally, tamely ramified extension of $\mathbb{Q}_{p_{j}}$. Thus $K_{\mathfrak{p}_{j}}=\mathbb{Q}_{p_{j}}\left(\sqrt[l]{p_{j} v_{j}}\right)$ with a unit $v_{j} \in \mathbb{Q}_{p_{j}}$. Abbreviate $\pi_{j}=\sqrt[l]{p_{j} v_{j}}$, so $\pi_{j}$ is a prime element in $K_{\mathfrak{p}_{j}}$, and write $\alpha=\pi_{j}^{a_{j}} \cdot v$ with a unit $v$. Then

$$
\begin{aligned}
\left(\alpha^{g_{0}-1}\right)^{c_{0 j}} & \equiv\left(\alpha^{g_{0}-1}\right)^{1+g_{0}+\ldots+g_{0}^{c_{0 j}-1}}=\alpha^{g_{0}^{c_{0 j}}-1}=\left(\pi_{j}^{a_{j}} v\right)^{g_{0}^{c_{0 j}}-1} \equiv\left(\pi_{j}^{a_{j}}\right)^{g_{0}^{c_{0 j}}-1} \\
& =\pi_{j}^{a_{j}\left(\left(q_{0}, K_{\mathfrak{p}_{j}} / \mathbb{Q}_{p_{j}}\right)-1\right)} \stackrel{(1)}{=}\left(p_{j} v_{j}, q_{0}\right)_{\mathbb{Q}_{p_{j}, l}}^{a_{j}}=\left(p_{j}, q_{0}\right)_{\mathbb{Q}_{p_{j}, l}}^{a_{j}}\left(v_{j}, q_{0}\right)_{\mathbb{Q}_{p_{j}, l}}^{a_{j}} \\
& \stackrel{(2)}{=} q_{0}^{-m_{j} a_{j}} \cdot 1,
\end{aligned}
$$

with the equality (1) and congruence (2) coming from [Se, pp. 215-217].

6. Euler systems. The purpose of this section is to recall some basic properties of Euler systems. The general reference is $[\mathrm{Ru}]$.

Let $K \subset \mathbb{Q}\left(\zeta_{n}\right)^{+}$, with $n$ denoting the conductor of $K$, and let $Q$ abbreviate squarefree products of rational primes $q$ splitting in $K$. For each such $q$ we fix a generator $\sigma_{q}$ of $\operatorname{Gal}\left(\mathbb{Q}\left(\zeta_{q}\right) / \mathbb{Q}\right)$ which whenever convenient is also regarded as a generator of the Galois group $G_{q}$ of the extension $K\left(\zeta_{q}\right) / K$. With this notation we have

$$
D_{q}:=\sum_{i=1}^{q-1} i \sigma_{q}^{i} \in \mathbb{Z}\left[G_{q}\right] \quad \text { satisfying } \quad\left(\sigma_{q}-1\right) D_{q}=q-1-\widehat{\sigma}_{q} .
$$

Set $D_{Q}=\prod_{q \mid Q} D_{q} \in \mathbb{Z}\left[G_{Q}\right]$ where $G_{Q}=\operatorname{Gal}\left(K\left(\zeta_{Q}\right) / K\right)$ is identified with $\prod_{q \mid Q} G_{q}$ in the usual way.

Our Euler system is $Q \mapsto \xi_{Q}=N_{\mathbb{Q}\left(\zeta_{n}, \zeta_{Q}\right) / K\left(\zeta_{Q}\right)}\left(1-\zeta_{n} \zeta_{Q}\right)$. It satisfies ES $1-4$ in $[\mathrm{Ru}]$. We now fix an odd prime $l \nmid n$ and a high power $L$ of it and use the notation $\mathfrak{b}_{1}={ }_{L} \mathfrak{b}_{2}$ for ideals $\mathfrak{b}_{1}, \mathfrak{b}_{2}$ of $K$ in order to indicate that $\mathfrak{b}_{1} \mathfrak{b}_{2}^{-1}$ is an $L$ th power of an ideal in $K$. In what follows the primes $q$ not only split in $K$ but also satisfy $q \equiv 1 \bmod L$.

Assume that $\alpha \in K^{\times}$is prime to $q$. Then there is a $\beta \in K\left(\zeta_{q}\right)^{\times}$such that $\alpha \equiv \beta^{1-\sigma_{q}} \bmod \mathfrak{Q}$ for all prime ideals $\mathfrak{Q} \mid q$ of $K\left(\zeta_{q}\right)$. We define the ideal $\varphi_{q}(\alpha)$ by

$$
\varphi_{q}(\alpha)={ }_{L} \prod_{\mathfrak{q} \mid q} \mathfrak{q}^{v_{\mathfrak{q}}(\beta)}\left({ }^{13}\right) .
$$

So $\varphi_{q}$ is a $\operatorname{Gal}(K / \mathbb{Q})$-homomorphism taking values in the ideals of $K$ sup-

$\left({ }^{13}\right) v_{\mathfrak{q}}(\beta)$ is the $\mathfrak{Q}$-value of $\beta$ for the unique prime $\mathfrak{Q} \mid \mathfrak{q}$ in $K\left(\zeta_{q}\right)$. 
ported in $q$, modulo $L$ th powers of ideals; it is in fact the precise analogue of the $\varphi_{q}$ in the situation of $[\mathrm{Ru}]$.

The following holds:

$$
\xi_{Q}^{D_{Q}(\sigma-1)} \text { is an } L \text { th power in } K\left(\zeta_{Q}\right) \text { for all } \sigma \in G_{Q} .
$$

$\sigma \mapsto \sqrt[L]{\xi_{Q}^{D_{Q}(\sigma-1)}}$ is a split 1-cocycle, so yields a unique $\kappa_{Q} \in$ $K^{\times} / K^{\times L}$ with $\kappa_{Q} \equiv \xi_{Q}^{D_{Q}} \bmod K\left(\zeta_{Q}\right)^{\times L}$.

As in $[\mathrm{Ru}]$, we set $\kappa_{1}=\xi_{1}$.

(6.5) The $q$-part $\left(\kappa_{Q}\right)_{q}$ in the principal ideal $\left(\kappa_{Q}\right)$ is

$$
={ }_{L} \begin{cases}(1) & \text { if } q \nmid Q, \\ \varphi_{q}\left(\kappa_{Q / q}\right) & \text { if } q \mid Q .\end{cases}
$$

(6.6) Set $G=\operatorname{Gal}(K / \mathbb{Q})$. Let $W$ be a finite $G$-submodule of $K^{\times} / K^{\times L}$ and $\psi: W \rightarrow \mathbb{Z} / L[G]$ a $G$-homomorphism. Then, to a given ideal class $\mathfrak{C}$ of $K$, there exist a unit $u \in(\mathbb{Z} / L)^{\times}$and infinitely many primes $\mathfrak{q}$ of $K$ such that $\mathfrak{q} \in \mathfrak{C}$, the rational prime $q \in \mathfrak{q}$ splits in $K$ and is $\equiv 1 \bmod L,(w)_{q}={ }_{L} 1$ and $\varphi_{q}(w)={ }_{L} \mathfrak{q}^{u \psi(w)}$ for all $w \in W$.

The following lemma concentrates on the unit $u$ in (6.6). Again, $G=$ $\operatorname{Gal}(K / \mathbb{Q})$.

Lemma 6.1. Let $q_{0}$ be a rational prime which is unramified in K. Assume that we are given a triple $L, W, \psi$ as in (6.6) with $q_{0} \in W, \psi\left(q_{0}\right)=\widehat{G}$ and that in accordance with these data and a given ideal class $\mathfrak{C}$ in $K$ a prime $\mathfrak{q}$ has been picked. Then

(i) $\left(q_{0}, \mathbb{Q}\left(\zeta_{q}\right) / \mathbb{Q}\right)$ generates $\operatorname{Gal}\left(\mathbb{Q}\left(\zeta_{q}\right) / \mathbb{Q}\right)$ modulo Lth powers.

(ii) If the restriction of $\sigma_{q}$ to $\mathbb{Q}\left(\zeta_{q}\right)$ and $\left(q_{0}, \mathbb{Q}\left(\zeta_{q}\right) / \mathbb{Q}\right)^{-1}$ differ by an Lth power, then the corresponding $\varphi_{q}$ satisfies $\varphi_{q}(w)={ }_{L} \mathfrak{q}^{\psi(w)}$ for all $w \in W$.

(iii) Assuming $\sigma_{q}$ chosen as in (ii) and $\psi(a)=t \widehat{G}$ for a rational $a \in W$, the automorphisms $\left.\sigma_{q}^{t}\right|_{\mathbb{Q}\left(\zeta_{q}\right)}$ and $\left(a, \mathbb{Q}\left(\zeta_{q}\right) / \mathbb{Q}\right)^{-1}$ differ by an Lth power.

Proof. For the chosen generator $\sigma_{q}$ of $G_{q}$ define $s \in \mathbb{Z} /(q-1)$ by

$$
\left(q_{0}, \mathbb{Q}\left(\zeta_{q}\right) / \mathbb{Q}\right)^{-1}=\left.\sigma_{q}^{s}\right|_{\mathbb{Q}\left(\zeta_{q}\right)} .
$$

Then $\varphi_{q}\left(q_{0}\right)={ }_{L} \mathfrak{q}^{u \psi\left(q_{0}\right)}=\mathfrak{q}^{u \widehat{G}}=\left(q^{u}\right)$ and so, by $(6.2)$,

$$
\prod_{\text {all } \mathfrak{q} \mid q} \mathfrak{q}^{v_{\mathfrak{q}}(\beta)}={ }_{L}\left(q^{u}\right) \quad \text { for any } \beta \in K\left(\zeta_{q}\right)^{\times}
$$

with $q_{0} \equiv \beta^{1-\sigma_{q}} \bmod \mathfrak{Q}$ for all $\mathfrak{Q} \mid q$. Now,

$$
\left(1-\zeta_{q}\right)^{\left(q_{0}, \mathbb{Q}\left(\zeta_{q}\right) / \mathbb{Q}\right)-1}=\frac{1-\zeta_{q}^{q_{0}}}{1-\zeta_{q}}=1+\zeta_{q}+\ldots+\zeta_{q}^{q_{0}-1} \equiv q_{0} \bmod \left(1-\zeta_{q}\right)
$$


implies

$$
\begin{aligned}
{\left[\left(1-\zeta_{q}\right)^{s}\right]^{1-\sigma_{q}} } & \equiv\left(1-\zeta_{q}\right)^{\left(1-\sigma_{q}\right)\left(1+\sigma_{q}+\ldots+\sigma_{q}^{s-1}\right)}=\left(1-\zeta_{q}\right)^{1-\sigma_{q}^{s}} \\
& =\left[\left(1-\zeta_{q}\right)^{\left(q_{0}, \mathbb{Q}\left(\zeta_{q}\right) / \mathbb{Q}\right)-1}\right]^{\left(q_{0}, \mathbb{Q}\left(\zeta_{q}\right) / \mathbb{Q}\right)^{-1}} \equiv q_{0} \bmod \left(1-\zeta_{q}\right) .
\end{aligned}
$$

Hence we may take $\beta=\left(1-\zeta_{q}\right)^{s}$ and obtain

$$
\left(q^{s}\right)=\prod_{\text {all } \mathfrak{q} \mid q} \mathfrak{q}^{s \cdot v_{\mathfrak{q}}\left(1-\zeta_{q}\right)}={ }_{L}\left(q^{u}\right)
$$

and then $s \equiv u \bmod L$. Thus $s$ is a unit $\bmod L$ and (i) is proved.

Letting $s^{\prime} \in(\mathbb{Z} /(q-1))^{\times}$satisfy $s^{\prime} \equiv s \bmod L$, we arrive at a new generator $\sigma_{q}^{\prime}=\sigma_{q}^{s^{\prime}}$ of $G_{q}$ and at a corresponding $\varphi_{q}^{\prime}$ with $\varphi_{q}^{\prime}\left(\alpha^{s^{\prime}}\right)=\varphi_{q}(\alpha)$ for all $\alpha \in K^{\times}$prime to $q$. Indeed, $\beta^{1-\sigma_{q}} \equiv \alpha \bmod \mathfrak{Q}$ implies $\beta^{1-\sigma_{q}^{\prime}}=$ $\left(\beta^{1-\sigma_{q}}\right)^{1+\sigma_{q}+\ldots+\sigma_{q}^{s^{\prime}-1}} \equiv\left(\beta^{1-\sigma_{q}}\right)^{s^{\prime}} \equiv \alpha^{s^{\prime}} \bmod \mathfrak{Q}$. Thus, for $w \in W, \varphi_{q}^{\prime}(w)^{s^{\prime}}$ $={ }_{L} \mathfrak{q}^{u \psi(w)}={ }_{L} \mathfrak{q}^{s^{\prime} \psi(w)}$, which gives (ii).

In order to see (iii) we go back to the beginning of our proof and replace $q_{0}$ by $a, s$ by $t^{\prime}$ and assume $u=1$. Then $\varphi_{q}(a)={ }_{L} \mathfrak{q}^{t \widehat{G}}=\left(q^{t}\right)$ and we conclude that $t^{\prime} \equiv t \bmod L$. Observe that (iii) implies that, modulo $q, a$ and $q_{0}^{t}$ only differ by an $L$ th power.

7. The case $r=2$. In this section we turn to the special case

$$
K \subset \mathbb{Q}\left(\zeta_{n}\right), \quad n=p_{1} p_{2}, \quad[K: \mathbb{Q}]=l \nmid n
$$

and prove conjecture (LC) as stated in Section 5 (and thus, at the same time, $(\mathrm{C})$ ). The notation is the one of the previous sections with

- $q_{0}$ chosen to simultaneously generate the Sylow $l$-subgroups of the multiplicative residue groups modulo $p_{1}$ and $p_{2}$, and to be inert in $K\left({ }^{14}\right)$,

- $L$ a power of $l$ which is greater than the power of $l$ in $p_{1}-1$ and $p_{2}-1$.

Set $W=\left\langle\alpha_{\infty}, q_{0}, p_{1}, p_{2}\right\rangle K^{\times L l^{h}}$ and let $\psi: W \rightarrow \mathbb{Z} / L l^{h}[G]$ be the $G$-map assigning $\alpha_{\infty}$ to $1, q_{0}$ to $\widehat{G}$ and $p_{j}$ to $t_{j} \widehat{G}(j=1,2)$ where the integers $t_{j}$ are chosen to satisfy

$$
t_{1} v_{\mathfrak{p}_{1}}\left(\alpha_{\infty}\right)+t_{2} v_{\mathfrak{p}_{2}}\left(\alpha_{\infty}\right) \equiv 1 \bmod L l^{h} .
$$

This congruence ensures that $\psi$ respects every relation $\alpha_{\infty}^{x} q_{0}^{z_{0}} p_{1}^{z_{1}} p_{2}^{z_{2}} \in K^{\times L l^{h}}$ with $x \in \mathbb{Z} G$ and integers $z_{0}, z_{1}, z_{2}$. In fact, first of all $\alpha_{\infty}^{\left(g_{0}-1\right) x}$ is an $L l^{h}$ th power in $K^{\times}$and, being a unit, therefore in $E_{K}$. Read in $\mathbb{Z}_{l} \otimes E_{K}$, the proof of Lemma 2.2 yields an $x_{1} \in \mathbb{Z}_{l} G$ such that $\alpha_{\infty}^{\left(g_{0}-1\right) x}=\alpha_{\infty}^{\left(g_{0}-1\right) x_{1} L l^{h}}$, i.e.,

$\left({ }^{14}\right)$ The compositum $\widehat{K}$ of the extensions $K_{j} \subset \mathbb{Q}\left(\zeta_{j}\right)$ of degree $l$ over $\mathbb{Q}$ contains a field $K_{0}$ of degree $l$ which is different from $K_{1}, K, K_{2}$ as $l \neq 2$, and we choose $q_{0}$ so that the Frobenius automorphism at $q_{0}$ generates $\operatorname{Gal}\left(\widehat{K} / K_{0}\right)$. 
$x-x_{1} L l^{h}=x_{2} \widehat{G}, x_{2} \in \mathbb{Z}_{l} G$. It follows that

$$
\alpha_{\infty}^{\widehat{G} x_{2}} q_{0}^{z_{0}} p_{1}^{z_{1}} p_{2}^{z_{2}}=p_{1}^{x_{2} v_{\mathfrak{p}_{1}}\left(\alpha_{\infty}\right)+z_{1}} p_{2}^{x_{2} v_{\mathfrak{p}_{2}}\left(\alpha_{\infty}\right)+z_{2}} q_{0}^{z_{0}} \in K^{\times L l^{h}} \cap \mathbb{Q}^{\times}=\mathbb{Q}^{\times L l^{h}},
$$

so $L l^{h}\left|z_{0}, L l^{h}\right| x_{2} v_{\mathfrak{p}_{j}}\left(\alpha_{\infty}\right)+z_{j}$ and the above relation is a consequence of

$$
\alpha_{\infty}^{\widehat{G}} p_{1}^{-v_{\mathfrak{p}_{1}}\left(\alpha_{\infty}\right)} p_{2}^{-v_{\mathfrak{p}_{2}}\left(\alpha_{\infty}\right)} \in K^{\times L l^{h}},
$$

which is respected by $\psi$.

In accordance with the data $L l^{h}, W$ and $\psi$ we employ (6.6) and choose a prime $\mathfrak{q}_{1} \in \mathfrak{C}_{1}$ so that $N_{K / \mathbb{Q}} \mathfrak{q}_{1}=q_{1} \equiv 1 \bmod L l^{h}$ splits in $K$ and $\left(\kappa_{q_{1}}\right)={ }_{L l^{h}}$ $\varphi_{q_{1}}\left(\kappa_{1}\right)={ }_{L l^{h}} \mathfrak{q}_{1}^{\psi\left(\kappa_{1}\right)}$, by (6.5) and Lemma 6.1 with an appropriate choice of $\sigma_{q_{1}}$.

Now, $\kappa_{1}=\xi_{1}=\alpha_{\infty}^{\left(g_{0}-1\right)^{h}}$. This is seen as follows. First, $\xi_{1}^{g_{0}-1}=\xi_{K}^{g_{0}-1}$ because

$$
\xi_{K}=\xi_{1} \cdot N_{\mathbb{Q}\left(\zeta_{n}\right) / K}\left(\left(1-\zeta_{n}^{p_{1}}\right)\left(1-\zeta_{n}^{p_{2}}\right)\right)
$$

with

$$
\begin{aligned}
N_{\mathbb{Q}\left(\zeta_{n}\right) / K}\left(1-\zeta_{n}^{p_{j}}\right) & =N_{\mathbb{Q}\left(\zeta_{n}\right) / K}\left(1-\zeta_{p_{j^{\prime}}}\right) \\
& =N_{K\left(\zeta_{p_{j^{\prime}}}\right) / K} N_{\mathbb{Q}\left(\zeta_{n}\right) / K\left(\zeta_{p_{j^{\prime}}}\right)}\left(1-\zeta_{p_{j^{\prime}}}\right)=p_{j^{\prime}}^{m_{j}}
\end{aligned}
$$

where $\left\{j, j^{\prime}\right\}=\{1,2\}$ and $m_{j}=\left(p_{j}-1\right) / l$. Second, by Lemma $2.2, \xi_{1}^{g_{0}-1}=$ $\alpha_{\infty}^{\left(g_{0}-1\right)^{h+1}}$, which gives the assertion as $\xi_{1}$ and $\alpha_{\infty}^{\left(g_{0}-1\right)^{h}}$ both have norm 1 .

We therefore get $\left(\kappa_{q_{1}}\right)=\mathfrak{q}_{1}^{\left(g_{0}-1\right)^{h}} \cdot \mathfrak{r}^{L l^{h}}$ for some ideal $\mathfrak{r}$. Since $\mathfrak{r}^{L l^{h}}$ is principal, $\mathfrak{r}^{l^{h}}$ is principal, $\mathfrak{r}^{l^{h}}=(\rho)$ say. Replacing $\kappa_{q_{1}}$ by $\kappa_{q_{1}} \rho^{-L}$ we may assume

$$
\left(\kappa_{q_{1}}\right)=\mathfrak{q}_{1}^{\left(g_{0}-1\right)^{h}} .
$$

It is important to note that this $\kappa_{q_{1}}$ is obtained as a splitting of a cocycle as in (6.4), although with $L$ and no longer with $L l^{h}$.

The element $\kappa_{q_{1}}$ has norm 1 in $\mathbb{Q}$ (after multiplying it with $-1=(-1)^{L}$ if necessary), so there exists an $\alpha_{\mathfrak{q}_{1}} \in K^{\times}$such that $\alpha_{\mathfrak{q}_{1}}^{g_{0}-1}=\kappa_{q_{1}}$. Then $\left(\alpha_{\mathfrak{q}_{1}}\right)=\mathfrak{q}_{1}^{\left(g_{0}-1\right)^{h-1}} \mathfrak{a}_{1}$ with $\mathfrak{a}_{1}$ (after multiplying $\alpha_{\mathfrak{q}_{1}}$ by a rational number) supported on $\mathfrak{p}_{1}, \mathfrak{p}_{2}$. The notation $\alpha_{\mathfrak{q}_{1}}$ can now be interpreted in the sense of Section 5.

Proposition 7.1. $\kappa_{q_{1}} \equiv q_{1}^{m_{j} t_{j^{\prime}}} \bmod \mathfrak{p}_{j}$, where $j^{\prime} \neq j$.

Recall that $m_{j}=\left(p_{j}-1\right) / l$. The proof of the proposition is delayed to the end of this section.

Because $\left(q_{1}, K_{\mathfrak{p}_{j}} / \mathbb{Q}_{p_{j}}\right)^{c_{0 j}}=\left(q_{0}, K_{\mathfrak{p}_{j}} / \mathbb{Q}_{p_{j}}\right)^{c_{1 j}}, q_{1}^{c_{0 j}}$ and $q_{0}^{c_{1 j}}$ differ by a norm, so by an $l$ th power modulo $p_{j}$, and we deduce $q_{1}^{m_{j} c_{0 j}} \equiv q_{0}^{m_{j} c_{1 j}} \bmod p_{j}$. Substituting this in Proposition 7.1 we have

$$
\alpha_{\mathfrak{q}_{1}}^{c_{0 j}\left(g_{0}-1\right)} \equiv q_{0}^{m_{j} t_{j^{\prime}} c_{1 j}} \bmod \mathfrak{p}_{j}
$$


We compare this congruence with the one given in Lemma 5.3:

$$
\alpha_{\mathfrak{q}_{1}}^{c_{0 j}\left(g_{0}-1\right)} \equiv q_{0}^{-m_{j} v_{\mathfrak{p}_{j}}\left(\alpha_{\mathfrak{q}_{1}}\right)} \bmod \mathfrak{p}_{j} .
$$

By the choice of $q_{0}, q_{0}^{m_{j}} \not \equiv 1 \bmod p_{j}$, whence

$$
-v_{\mathfrak{p}_{j}}\left(\alpha_{\mathfrak{q}_{1}}\right) \equiv t_{j^{\prime}} c_{1 j} \bmod l .
$$

The reciprocity law gives

$$
\sum_{j=1}^{2} c_{i j} \equiv \begin{cases}-1, & i=0 \\ 0, & i \neq 0\end{cases}
$$

thus

$$
-c_{12} \equiv \operatorname{det}\left(\begin{array}{cc}
c_{01} & c_{02} \\
c_{11} & c_{12}
\end{array}\right) \equiv c_{11} \bmod l
$$

and

$$
\begin{aligned}
\operatorname{det}\left(\begin{array}{cc}
-v_{\mathfrak{p}_{1}}\left(\alpha_{\infty}\right) & -v_{\mathfrak{p}_{2}}\left(\alpha_{\infty}\right) \\
-v_{\mathfrak{p}_{1}}\left(\alpha_{\mathfrak{q}_{1}}\right) & -v_{\mathfrak{p}_{2}}\left(\alpha_{\mathfrak{q}_{1}}\right)
\end{array}\right) & \equiv \operatorname{det}\left(\begin{array}{cc}
-v_{\mathfrak{p}_{1}}\left(\alpha_{\infty}\right) & -v_{\mathfrak{p}_{2}}\left(\alpha_{\infty}\right) \\
t_{2} c_{11} & t_{1} c_{12}
\end{array}\right) \\
& \equiv c_{11} \operatorname{det}\left(\begin{array}{cc}
-v_{\mathfrak{p}_{1}}\left(\alpha_{\infty}\right) & -v_{\mathfrak{p}_{2}}\left(\alpha_{\infty}\right) \\
t_{2} & -t_{1}
\end{array}\right) \\
& \equiv c_{11}\left(t_{1} v_{\mathfrak{p}_{1}}\left(\alpha_{\infty}\right)+t_{2} v_{\mathfrak{p}_{2}}\left(\alpha_{\infty}\right)\right) \\
& \equiv c_{11} \equiv \operatorname{det}\left(\begin{array}{cc}
c_{01} & c_{02} \\
c_{11} & c_{12}
\end{array}\right) \bmod l
\end{aligned}
$$

which proves (LC).

Proof (of Proposition 7.1). Fix a generator $\sigma_{q_{1}}$ of $G_{q_{1}}$, as in Lemma 6.1(ii). Then $\left.\sigma_{q_{1}}^{\tilde{t}_{j}}\right|_{\mathbb{Q}\left(\zeta_{q_{1}}\right)}=\left(p_{j}, \mathbb{Q}\left(\zeta_{q_{1}}\right) / \mathbb{Q}\right)^{-1}$ for a unique $\widetilde{t}_{j} \bmod \left(q_{1}-1\right)$ and we have $\widetilde{t}_{j} \equiv t_{j} \bmod L$ by Lemma $6.1(\mathrm{iii})$. The Euler system in Section 6 has

$$
\begin{aligned}
\xi_{q_{1}} & =N_{\mathbb{Q}\left(\zeta_{n}, \zeta_{q_{1}}\right) / K\left(\zeta_{q_{1}}\right)}\left(1-\zeta_{n} \zeta_{q_{1}}\right) \\
& \equiv N_{\mathbb{Q}\left(\zeta_{n}, \zeta_{q_{1}}\right) / K\left(\zeta_{q_{1}}\right)}\left(1-\zeta_{p_{j^{\prime}}} \zeta_{q_{1}}\right) \bmod \left(1-\zeta_{p_{j}}\right) \\
& =N_{K\left(\zeta_{p_{j^{\prime}}}, \zeta_{q_{1}}\right) / K\left(\zeta_{q_{1}}\right)} N_{\mathbb{Q}\left(\zeta_{n}, \zeta_{q_{1}}\right) / K\left(\zeta_{p_{j^{\prime}}}, \zeta_{q_{1}}\right)}\left(1-\zeta_{p_{j^{\prime}}} \zeta_{q_{1}}\right) \\
& =N_{K\left(\zeta_{p_{j^{\prime}}}, \zeta_{q_{1}}\right) / K\left(\zeta_{q_{1}}\right)}\left(1-\zeta_{p_{j^{\prime}}} \zeta_{q_{1}}\right)^{m_{j}} \\
& =\left(\frac{1-\zeta_{q_{1}}^{p_{j^{\prime}}}}{1-\zeta_{q_{1}}}\right)^{m_{j}}=\left(1-\zeta_{q_{1}}\right)^{m_{j}\left(\left(p_{j^{\prime}}, \mathbb{Q}\left(\zeta_{q_{1}}\right) / \mathbb{Q}\right)-1\right)} \\
& =\left(1-\zeta_{q_{1}}\right)^{m_{j}\left(\sigma_{q_{1}}^{-\tilde{t}_{j^{\prime}}}-1\right)}
\end{aligned}
$$

and thus

$$
\xi_{q_{1}} \equiv\left(1-\zeta_{q_{1}}\right)^{m_{j}\left(\sigma_{q_{1}}-1\right)\left(1+\sigma_{q_{1}}+\ldots+\sigma_{q_{1}}^{-\tilde{t}_{j^{\prime}}-1}\right)} \bmod \left(1-\zeta_{p_{j}}\right)
$$


On applying $D_{q_{1}}$ this implies, by (6.1),

$$
\begin{aligned}
& \xi_{q_{1}}^{D_{q_{1}}} \equiv\left(1-\zeta_{q_{1}}\right)^{m_{j}\left(1+\sigma_{q_{1}}+\ldots+\sigma_{q_{1}}^{-\tilde{t}_{j^{\prime}}-1}\right)\left(q_{1}-1-\widehat{\sigma}_{q_{1}}\right)} \\
& =\left(1-\zeta_{q_{1}}\right)^{m_{j}\left(q_{1}-1\right)\left(1+\sigma_{q_{1}}+\ldots+\sigma_{q_{1}}^{-\tilde{t}_{j^{\prime}}-1}\right)} q_{1}^{-m_{j}\left(1+\sigma_{q_{1}}+\ldots+\sigma_{q_{1}}^{-\tilde{t}_{j^{\prime}}-1}\right)} \\
& \equiv\left(1-\zeta_{q_{1}}\right)^{m_{j}\left(q_{1}-1\right)\left(1+\sigma_{q_{1}}+\ldots+\sigma_{q_{1}}^{-\tilde{t}_{j^{\prime}}-1}\right)} \cdot q_{1}^{m_{j} t_{j^{\prime}}} \bmod \left(1-\zeta_{p_{j}}\right) \text {. }
\end{aligned}
$$

By (6.4), with $\beta_{q_{1}} \in K\left(\zeta_{q_{1}}\right)$ satisfying $\beta_{q_{1}}^{\sigma-1}=\sqrt[L]{\xi_{q_{1}}^{D_{q_{1}}(\sigma-1)}}$ we have

$$
\kappa_{q_{1}}=\xi_{q_{1}}^{D_{q_{1}}} / \beta_{q_{1}}^{L} .
$$

Because of $\left(\kappa_{\mathfrak{q}_{1}}\right)=\mathfrak{q}_{1}^{\left(g_{0}-1\right)^{h}}$ and since $\xi_{q_{1}}$ is a unit, it follows that $\beta_{q_{1}}$ is supported in the orbit of $\mathfrak{q}_{1}$ (in $K\left(\zeta_{q_{1}}\right)$ ). And we have $\xi_{q_{1}}^{\widehat{\sigma}_{q_{1}}}={ }_{\mathrm{ES} 3} \xi_{1}^{\operatorname{Frob}\left(q_{1}\right)-1}$ $=1$, as $q_{1}$ splits in $K$.

Thus $\xi_{q_{1}}^{D_{q_{1}}\left(\sigma_{q_{1}}-1\right)}=\xi_{q_{1}}^{q_{1}-1-\widehat{\sigma}_{q_{1}}}=\xi_{q_{1}}^{q_{1}-1}$ and so $\beta_{q_{1}}^{\sigma_{q_{1}}-1}=\xi_{q_{1}}^{\left(q_{1}-1\right) / L}$ which, by (7.1), gives

$$
\beta_{q_{1}}^{\sigma_{q_{1}}-1} \equiv\left(1-\zeta_{q_{1}}\right)^{\frac{q_{1}-1}{L} m_{j}\left(1+\sigma_{q_{1}}+\ldots+\sigma_{q_{1}}^{-\tilde{t}_{j^{\prime}}-1}\right)\left(\sigma_{q_{1}}-1\right)} \bmod \left(1-\zeta_{p_{j}}\right) .
$$

Since $\beta_{q_{1}}$ and $1-\zeta_{q_{1}}$ are in $K\left(\zeta_{q_{1}}\right)$ this congruence can be read modulo the product $\widetilde{\mathfrak{p}}_{j}$ of the primes of $K\left(\zeta_{q_{1}}\right)$ above $\mathfrak{p}_{j}$, which implies

$$
\beta_{q_{1}} \equiv\left(1-\zeta_{q_{1}}\right)^{\frac{q_{1}-1}{L}} m_{j}\left(1+\sigma_{q_{1}}+\ldots+\sigma_{q_{1}}^{-\tilde{t}_{j^{\prime}}-1}\right) \gamma_{q_{1}} \bmod \widetilde{\mathfrak{p}}_{j}
$$

with $\gamma_{q_{1}}$ in $K\left(\zeta_{q_{1}}\right)$ so that $\gamma_{q_{1}}^{\sigma_{q_{1}}-1} \equiv 1 \bmod \tilde{\mathfrak{p}}_{j}$. As $\mathfrak{p}_{j}$ is unramified in $K\left(\zeta_{q_{1}}\right)$ this means that $\gamma_{q_{1}}$ may be taken to be in $K$, hence

$$
\gamma_{q_{1}}^{p_{j}-1} \equiv 1 \bmod \mathfrak{p}_{j}
$$

Therefore, by (7.3), (7.2), (7.4),

$$
\kappa_{q_{1}} \equiv q_{1}^{m_{j} t_{j^{\prime}}} \cdot \gamma_{q_{1}}^{-L} \bmod \mathfrak{p}_{j}
$$

Since $\kappa_{q_{1}}$ has norm 1 , its $l$ th power is congruent to 1 modulo $\mathfrak{p}_{j}$, as is the $l$ th power of $q_{1}^{m_{j} t_{j^{\prime}}}$. So $\gamma_{q_{1}}^{L l} \equiv 1 \bmod \mathfrak{p}_{j}$. By (7.5) and the choice of $L$ thus $\gamma_{q_{1}}^{L} \equiv 1 \bmod \mathfrak{p}_{j}$. This finishes the proof.

\section{References}

[Bu] D. Burns, Equivariant Tamagawa numbers and Galois module theory, I, preprint, King's College London, 1997.

[Cc] C. Chevalley, Sur la théorie du corps de classes dans les corps finis et les corps locaux, J. Fac. Sci. Tokyo 2 (1933), 365-476.

[Ct] T. Chinburg, On the Galois structure of algebraic integers and S-units, Invent. Math. 74 (1983), 321-349. 
[Fr] A. Fröhlich, Central Extensions, Galois Groups, and Ideal Class Groups of Number Fields, Contemp. Math. 24, Amer. Math. Soc., 1983.

[GRW1] K. W. Gruenberg, J. Ritter and A. Weiss, A local approach to Chinburg's Root Number Conjecture, Proc. London Math. Soc. 79 (1999), 47-80.

[GRW2] - - - - On Chinburg's Root Number Conjecture, Jahresber. Deutsch. Math.Verein. 100 (1998), 36-44.

[La] S. Lang, Cyclotomic Fields I and II, Grad. Texts in Math. 121, Springer, New York, 1990.

[RW] J. Ritter and A. Weiss, Cohomology of units and L-values at zero, J. Amer. Math. Soc. 10 (1997), 513-552.

[Ru] K. Rubin, The Main Conjecture, Appendix in [La].

[Se] J. P. Serre, Corps Locaux, Hermann, Paris, 1968.

[Sn] V. Snaith, Galois Module Structure, Fields Inst. Monogr. 2, Amer. Math. Soc., 1994.

[Ta] J. Tate, Les Conjectures de Stark sur les Fonctions L d'Artin en $s=0$, Progr. Math. 47, Birkhäuser, 1984.

[Wa] L. Washington, Introduction to Cyclotomic Fields, Grad. Texts in Math. 83, Springer, 1982.

[We] A. Weiss, Multiplicative Galois Module Structure, Fields Inst. Monogr. 5, Amer. Math. Soc., 1996.

Institut für Mathematik

Universität Augsburg

D-86135 Augsburg, Germany
Department of Mathematics

University of Alberta

Edmonton T6G 2G1, Canada 